\title{
Characterization of surjective partial differential operators on spaces of real analytic functions
}

\author{
by \\ Michael Langenbruch (Oldenburg) \\ Dedicated to Professor D. Vogt on the occasion of his 60th birthday
}

\begin{abstract}
Let $A(\Omega)$ denote the real analytic functions defined on an open set $\Omega \subset \mathbb{R}^{n}$. We show that a partial differential operator $P(D)$ with constant coefficients is surjective on $A(\Omega)$ if and only if for any relatively compact open $\omega \subset \Omega, P(D)$ admits (shifted) hyperfunction elementary solutions on $\Omega$ which are real analytic on $\omega$ (and if the equation $P(D) f=g, g \in A(\Omega)$, may be solved on $\omega)$. The latter condition is redundant if the elementary solutions are defined on $\operatorname{conv}(\Omega)$. This extends and improves previous results of Andersson, Kawai, Kaneko and Zampieri. For convex $\Omega$, a different characterization of surjective operators $P(D)$ on $A(\Omega)$ was given by Hörmander using a Phragmén-Lindelöf type condition, which cannot be extended to the case of noncovex $\Omega$. The paper is based on a surjectivity criterion for exact sequences of projective (DFS)spectra which improves earlier results of Braun and Vogt, and Frerick and Wengenroth.
\end{abstract}

This paper is concerned with the basic question when

$$
P(D): A(\Omega) \rightarrow A(\Omega) \text { is surjective. }
$$

Here $P(D)$ is a partial differential operator with constant coefficients, $\Omega \subset$ $\mathbb{R}^{n}$ is an open set and $A(\Omega)$ is the space of real analytic functions on $\Omega$.

Several methods have been developed to solve this problem: The first counterexample to $(0.1)$ is due to Piccinini [34] who showed that the heat equation is not surjective on $A\left(\mathbb{R}^{3}\right)$ (see also the conjecture of De Giorgi and Cattabriga [10]). Then Hörmander [13] characterized (0.1) for convex sets $\Omega$ by means of a Phragmén-Lindelöf condition valid on the complex variety of $P$. Since then Hörmander's method has been adapted by several authors for further studies on this problem (Miwa [31], Andreotti and Nacinovich [3], Zampieri [42], Braun [6] and the recent series of papers of Braun, Meise and Taylor $[7,8])$.

2000 Mathematics Subject Classification: Primary 35E10, 35E05; Secondary 46F15.

Key words and phrases: partial differential operator, real analytic function, elementary solution, hyperfunctions, analytic singular support. 
Hörmander's criterion is restricted to convex sets $\Omega$ by the use of Fourier theory. On the other hand, using hyperfunctions and so-called "good elementary solutions" for $P(D)$, Kawai [18] was able to prove ( 0.1$)$ for locally hyperbolic operators on special, not necessarily convex bounded open sets $\Omega$. The assumption of boundedness was removed by Kaneko [15] (see also Kaneko $[16,17])$. Andersson [2] used a similar idea to show that locally hyperbolic operators are surjective on $A\left(\mathbb{R}^{n}\right)$.

While Kawai mainly ignored the (complicated) topology of $A(\Omega)$, Hörmander observed that the problem is intimately related to a "decomposition with bounds" in the kernel of $P(D)$. In the present paper we will use the theory of $\mathrm{Proj}^{k}$-functors of Palamodov [32,33] (see also Vogt [38]), which is an abstract version of Hörmander's observation, to prove the following characterization of (0.1) for general open sets $\Omega$ in the spirit of Kawai's work: Let $\mathfrak{B}(\Omega)$ denote the hyperfunctions on $\Omega$ and for $\delta>0$ let

$$
A_{\delta}(\omega):=\left\{f \in A(\omega)\left|\sup _{x \in \omega}\right| f^{(a)}(x) \mid \delta^{|a|} / a !<\infty\right\} .
$$

TheOREM. Let $\Omega \subset \mathbb{R}^{n}$ be open. The following statements are equivalent:

(a) $P(D): A(\Omega) \rightarrow A(\Omega)$ is surjective.

(b) (i) For any $g \in A(\Omega)$ and any $\omega \subset \subset \Omega$ there is $f \in A(\omega)$ such that

$$
P(D) f=\left.g\right|_{\omega} .
$$

(ii) $P(D)$ satisfies the following condition $\left(\underline{\mathrm{B}_{\Omega}}\right)$ : for any $\omega \subset \subset \Omega$ there is $\widetilde{\omega} \subset \subset \Omega$ with $\widetilde{\omega} \supset \supset \omega$ such that $\overline{\text { for }}$ any $\xi \in \partial \widetilde{\omega}$ and any $\widehat{\omega} \subset \subset \Omega$ there is $F \in \mathfrak{B}(\widehat{\omega})$ such that

$$
P(D) F=\delta_{\xi} \quad \text { on } \widehat{\omega} \quad \text { and }\left.\quad F\right|_{\omega} \in A(\omega),
$$

where $\delta_{\xi}$ is the point evaluation at $\xi$.

(c) $P(D)$ satisfies $(\mathrm{b})(\mathrm{i})$ and the following condition $\left(\overline{\mathrm{B}_{\Omega}}\right)$ : for any $\omega \subset \subset$ $\Omega$ there are $\widetilde{\omega} \subset \subset \Omega$ and $\delta>0$ such that for any $\xi \in \Omega \backslash \widetilde{\omega}$ there is $F \in \mathfrak{B}(\Omega)$ such that

$$
P(D) F=\delta_{\xi} \quad \text { on } \Omega \quad \text { and }\left.\quad F\right|_{\omega} \in A_{\delta}(\omega) .
$$

This result is part of our Main Theorem 2.1 where further equivalent characterizations of (0.1) are given (including the important technical condition $\left.\left(\underline{\mathrm{A}_{\Omega}}\right)\right)$.

$\left(\overline{\mathrm{B}_{\Omega}}\right)$ is similar to the criterion for surjectivity of $P(D)$ on nonquasianalytic classes of ultradifferentiable functions of Roumieu type which we proved in Langenbruch [22] (see also Langenbruch [23, 25]).

$\left(\overline{\mathrm{B}_{\Omega}}\right)$ improves the necessary condition for $(0.1)$ from Langenbruch $[24$, Theorem 1.3]. We used the latter condition to show that local hyperbolicity 
of the principal part $P_{m}$ of $P$ and hyperbolicity of the localizations of $P_{m}$ at $\infty$ are necessary for (0.1) in many situations (see Langenbruch [24, 26]).

The semiglobal solvability condition (0.2) is always satisfied if $\Omega$ is convex. Therefore, $(0.1)$ is equivalent to $\left(\underline{\mathrm{B}_{\Omega}}\right)$ and to $\left(\overline{\mathrm{B}_{\Omega}}\right)$ if $\Omega$ is convex.

$(0.2)$ is also redundant if the shifted elementary solutions $F$ are defined on convex sets. This gives the following two sufficient criteria:

$P(D)$ is surjective on $A(\Omega)$ if one of the following assumptions holds:

(i) For any $\omega \subset \subset \Omega$ there is $\widetilde{\omega} \subset \subset \Omega$ with $\omega \subset \subset \widetilde{\omega}$ such that for any $\widehat{\omega} \subset \subset \Omega$ and any $\xi \in \partial \widetilde{\omega}$ there is $F \in \mathfrak{B}(\operatorname{conv}(\widehat{\omega}))$ such that

$$
P(D) F=\delta_{\xi} \quad \text { on } \operatorname{conv}(\widehat{\omega}) \text { and }\left.F\right|_{\omega} \in A(\omega) .
$$

(ii) $P(D)$ satisfies $\left(\underline{\mathrm{B}_{\mathbb{R}^{n}}}\right)$ and for any $\omega \subset \subset \Omega$ and any $\zeta \in \partial \Omega$ there are a convex neighbourhood $U$ of $\bar{\Omega}$ and $F \in \mathfrak{B}(U)$ such that

$$
P(D) F=\delta_{\xi} \quad \text { on } U \text { and }\left.\quad F\right|_{\omega} \in A(\omega) .
$$

This contains and improves the corresponding results of Andersson [2], Kawai [18], Kaneko [15, 16] and Zampieri [42]. In particular, we get the following result for operators with locally hyperbolic principal part $P_{m}$ (see Section 2 for the definitions):

$P(D)$ is surjective on $A(\Omega)$ if for any $\zeta \in \partial \Omega$ and any $\Theta \in S^{n-1}$ there is $N_{\Theta, \zeta} \in S^{n-1}$ such that $P_{m}$ is locally hyperbolic with respect to $N_{\Theta, \zeta}$ and

$$
\left(\zeta+K\left(\left(P_{m}\right)_{\Theta}, N_{\Theta, \zeta}\right)\right) \cap \Omega=\emptyset,
$$

where $K\left(\left(P_{m}\right)_{\Theta}, N_{\Theta, \zeta}\right)$ is the local propagation cone of $P_{m}$ at $\Theta$ with respect to $N_{\Theta, \xi}$.

The paper is organized as follows: In the first section, a short introduction to the theory of the Proj ${ }^{k}$-functors of Palamodov (see Palamodov [32, 33] and Vogt [38]) is given. We here prove a strong surjectivity criterion for exact sequences of projective (DFS)-spectra (see Theorem 1.5) which improves the corresponding results of Braun and Vogt [9], Frerick and Wengenroth [11] and Wengenroth [39]: We will show that a continuous linear mapping is surjective if the kernel spectrum satisfies property $\left(\mathrm{P}_{3}\right)$ (instead of the apparently stronger condition $\left(\mathrm{P}_{2}\right)$ used in loc. cit.) and a suitable notion of reducedness. Moreover, we obtain a priori bounds for the solutions. In our concrete situation this implies that the following is also equivalent to the surjectivity of $P(D)$ on $A(\Omega)$ (see Theorem 2.1): for any neighbourhood $V \subset$ $\mathbb{C}^{n}$ of $\Omega$ there is a neighbourhood $W \subset \mathbb{C}^{n}$ of $\Omega$ such that $P(D) f=g$ may be solved with $f \in H(W)$ if $g \in H(V)$. Hörmander [13] proved such a priori Cauchy estimates for convex $\Omega$ using his Phragmén-Lindelöf principle.

In Section 2, the main result is stated in Theorem 2.1. We then prove the above consequences and discuss their relation to the literature and some examples. In Sections 3 and 4 we use the Grothendieck-Tillmann duality to 
show that the technical conditions $\left(\overline{\mathrm{A}_{\Omega}}\right)$ (and $\left(\underline{\mathrm{A}_{\Omega}}\right)$ ) are necessary (and sufficient, respectively) for (0.1). These are needed to prove the hyperfunction criteria $\left(\underline{\mathrm{B}_{\Omega}}\right)$ and $\left(\overline{\mathrm{B}_{\Omega}}\right)$ for $(0.1)$ in Section 5 .

The author wants to thank D. Vogt (Wuppertal) and P. Domański (Poznań) for several valuable discussions concerning the subject of this paper.

1. Projective (DFS)-spectra. We start with some useful notations and conventions: In this paper, $n \in \mathbb{N}$ is always at least 2 . The sets $\Omega$ and $\omega$ are always open in $\mathbb{R}^{n}$. We also assume that $\Omega$ is connected.

The real analytic functions on $\Omega$ are denoted by $A(\Omega)$. By $P(D)$ we always mean a partial differential operator in $n$ variables with constant coefficients. We are concerned with the question when

$$
P(D): A(\Omega) \rightarrow A(\Omega) \text { is surjective. }
$$

In this paper, we will extend real analytic functions on $\Omega$ to harmonic functions in $n+1$ variables defined near $\Omega$ in $\mathbb{R}^{n+1}$ (rather than to holomorphic functions defined near $\Omega$ in $\mathbb{C}^{n}$ ). To be precise, a point in $\mathbb{R}^{n+1}$ is usually written as $(x, y) \in \mathbb{R}^{n} \times \mathbb{R}$. As usual, $\Delta=\sum_{k \leq n}\left(\partial / \partial x_{k}\right)^{2}+(\partial / \partial y)^{2}$ denotes the Laplace operator on $\mathbb{R}^{n+1}$. The harmonic germs near a set $S \subset \mathbb{R}^{n+1}$ are denoted by $C_{\Delta}(S)$. It is clear that (1.1) holds if and only if

$$
P\left(D_{x}\right): C_{\Delta}(\Omega \times\{0\}) \rightarrow C_{\Delta}(\Omega \times\{0\}) \text { is surjective. }
$$

Indeed, the equivalence of (1.1) and (1.2) is obtained by means of the Cauchy problem for $\Delta$ with data on $\Omega \times\{0\} . C_{\Delta}(\Omega \times\{0\})$ is the projective limit of the projective spectrum

$$
C_{\Delta}^{\Omega}:=\left(C_{\Delta}\left(K_{k} \times\{0\}\right), R_{j}^{k}\right),
$$

where $\left\{K_{k} \mid k \in \mathbb{N}\right\}$ is an increasing compact exhaustion of $\Omega$, that is,

$$
K_{k} \subset \subset \operatorname{int}\left(K_{k+1}\right) \text { for each } k \text { and } \bigcup_{k=1}^{\infty} K_{k}=\Omega .
$$

The linking maps

$$
R_{j}^{k}: C_{\Delta}\left(K_{j} \times\{0\}\right) \rightarrow C_{\Delta}\left(K_{k} \times\{0\}\right) \quad \text { for } j \geq k
$$

are defined by restriction.

The theory of projective spectra of linear spaces and the corresponding $\mathrm{Proj}^{k}$-functors were developed by Palamodov [32, 33] (see also Vogt [38]). We will briefly introduce the corresponding notions and facts which we need. The reader is referred to these papers for further information.

For $S \subset \mathbb{R}^{n+1}$ let

$$
N_{P}(S):=\left\{f \in C_{\Delta}(S) \mid P\left(D_{x}\right) f=0\right\}
$$


and let

$$
N_{P}^{\Omega}:=\left(N_{P}\left(K_{k} \times\{0\}\right), R_{j}^{k}\right)
$$

be the projective spectrum of kernels of $P\left(D_{x}\right)$. We thus have the short sequence of projective spectra

$$
0 \rightarrow N_{P}^{\Omega} \rightarrow C_{\Delta}^{\Omega} \stackrel{P(D)}{\longrightarrow} C_{\Delta}^{\Omega} \rightarrow 0 .
$$

It is called exact if for any $k \in \mathbb{N}$ there is $j \geq k$ such that

$$
P(D) C_{\Delta}\left(K_{k} \times\{0\}\right) \supset R_{j}^{k}\left(C_{\Delta}\left(K_{j} \times\{0\}\right)\right) .
$$

We now have the following key result, which is essentially Theorem 5.1 of Vogt [38] in our concrete situation.

Proposition 1.1. Let the sequence (1.3) of projective spectra be exact. Then

$$
P(D): A(\Omega) \rightarrow A(\Omega) \text { is surjective }
$$

if and only if $\operatorname{Proj}^{1}\left(N_{P}^{\Omega}\right)=0$.

Proof. Necessity. Since (1.3) is exact, we have the exact sequence of linear spaces

$$
\begin{aligned}
0 & \rightarrow \operatorname{Proj}^{0}\left(N_{P}^{\Omega}\right) \rightarrow \operatorname{Proj}^{0}\left(C_{\Delta}^{\Omega}\right) \stackrel{P(D)}{\longrightarrow} \operatorname{Proj}^{0}\left(C_{\Delta}^{\Omega}\right) \\
& \rightarrow \operatorname{Proj}^{1}\left(N_{P}^{\Omega}\right) \rightarrow \operatorname{Proj}^{1}\left(C_{\Delta}^{\Omega}\right) \rightarrow \operatorname{Proj}^{1}\left(C_{\Delta}^{\Omega}\right) \rightarrow 0
\end{aligned}
$$

by Palamodov [32, p. 542]. We can identify $\operatorname{Proj}^{0}\left(N_{P}^{\Omega}\right)$ with $N_{P}(\Omega \times\{0\})$ (and $\operatorname{Proj}^{0}\left(C_{\Delta}^{\Omega}\right)$ with $C_{\Delta}(\Omega \times\{0\})$ ). Since $A(\Omega)$ (and hence $C_{\Delta}(\Omega \times\{0\})$ ) is ultrabornological by Martineau [29], Theorem 3.5 of Wengenroth [39] implies that

$$
\operatorname{Proj}^{1}\left(C_{\Delta}^{\Omega}\right)=0 .
$$

By (1.5) and (1.6) we have the exact sequence of linear spaces

$$
0 \rightarrow N_{p}(\Omega \times\{0\}) \rightarrow C_{\Delta}(\Omega \times\{0\}) \stackrel{P(D)}{\longrightarrow} C_{\Delta}(\Omega \times\{0\}) \rightarrow \operatorname{Proj}^{1}\left(N_{P}^{\Omega}\right) \rightarrow 0 .
$$

Since $P(D)\left(C_{\Delta}(\Omega \times\{0\})\right)=C_{\Delta}(\Omega \times\{0\})$ by the assumption and (1.2), this implies that $\operatorname{Proj}^{1}\left(N_{P}^{\Omega}\right)=0$.

Sufficiency. By (1.5) we get the exact sequence of linear spaces

$$
0 \rightarrow \operatorname{Proj}^{0}\left(N_{P}^{\Omega}\right) \rightarrow \operatorname{Proj}^{0}\left(C_{\Delta}^{\Omega}\right) \stackrel{P(D)}{\longrightarrow} \operatorname{Proj}^{0}\left(C_{\Delta}^{\Omega}\right) \rightarrow 0
$$

since $\operatorname{Proj}^{1}\left(N_{P}^{\Omega}\right)=0$ by assumption. The claim now follows by the above identification.

The exactness of the sequence (1.3) of spectra means that the equation $P(D) f=g$ can be solved semiglobally in $C_{\Delta}(\Omega)$. If $\Omega$ is convex, this semiglobal solvability easily follows from the solvability theory of (overdetermined) systems of partial differential equations. For general open sets $\Omega$, 
however, it must be proved before Proposition 1.1 can be used (see Proposition 3.3).

The reader is referred to Palamodov $[32,33]$ and Vogt [38] for the definition of the Proj ${ }^{1}$-functor. We do not need the definition here since we will only use explicit criteria for the vanishing of the $\mathrm{Proj}^{1}$-functor of projective (DFS)-spectra (see Theorems 1.2 and 1.5 below). We briefly introduce the corresponding notions and results in the form needed in this paper: Let $\mathfrak{X}=\left(X_{k}, R_{j}^{k}\right)$ be a projective (DFS)-spectrum, that is, a projective spectrum of (DFS)-spaces $X_{k}=\operatorname{limind}_{j \rightarrow \infty} X_{k, j}$ with Banach spaces $X_{k, j}$ and compact inclusion mappings from $X_{k, j}$ into $X_{k, j+1}$. Let $B_{k, j}$ be the unit ball in $X_{k, j}$. For $X:=\operatorname{limproj}_{k \rightarrow \infty} X_{k}$ let $R^{k}: X \rightarrow X_{k}$ be the canonical mapping.

A characterization of $\operatorname{Proj}^{1}(\mathfrak{X})=0$ has been obtained by Retakh [35]. We only need the necessity of a part of his criterion here:

Theorem 1.2 (Retakh [35, Theorem 3]). Let $\mathfrak{X}$ be a projective (DFS)spectrum. If $\operatorname{Proj}^{1}(\mathfrak{X})=0$, then for any $k \in \mathbb{N}$ there is $j \in \mathbb{N}$ with $j \geq k$ such that

$$
R_{j}^{k}\left(X_{j}\right) \subset R^{k}(X)+B_{k, j} .
$$

In our concrete situation we have the following:

$$
\mathfrak{X}:=N_{P}^{\Omega}=\left(N_{P}\left(K_{k} \times\{0\}\right), R_{j}^{k}\right)
$$

and

$$
N_{P}\left(K_{k} \times\{0\}\right):=\operatorname{limind}_{j \rightarrow \infty} N B_{P}\left(\left(K_{k} \times\{0\}\right)+V_{1 / j}\right),
$$

where $V_{\varepsilon}:=\left\{z \in \mathbb{R}^{n+1}|| z \mid<\varepsilon\right\}$ and for open $V \subset \mathbb{R}^{n+1}, N B_{P}(V)$ is the Banach space

$$
N B_{P}(V):=\left\{f \in N_{P}(V) \mid f \text { is bounded on } V\right\} .
$$

Our sufficient criterion for $\operatorname{Proj}^{1}(\mathfrak{X})=0$ is a variant of the results of Braun and Vogt [9], Frerick and Wengenroth [11] and Wengenroth [39] (see Theorem 1.5 below). There are two major differences as compared with these papers: Firstly, we will use condition $\left(\mathrm{P}_{3}\right)$ defined for the spectrum $\mathfrak{X}=\left(X_{k}, R_{j}^{k}\right)$ as follows:

$$
\forall \mu \exists k \forall K \exists n \forall m \exists N, S: R_{k}^{\mu}\left(B_{k, m}\right) \subset S\left(R_{K}^{\mu}\left(B_{K, N}\right)+B_{\mu, n}\right) .
$$

Notice that the order of quantifiers for $K$ and $n$ has been changed compared with condition $\left(\mathrm{P}_{2}\right)$ which has been used in loc. cit. This weaker order of quantifiers has apparently been overlooked, though the proofs of the corresponding results from [9] needed here can be applied almost without change. The use of $\left(\mathrm{P}_{3}\right)$ is essential to obtain the sufficiency of the criterion (e)(ii) of the Main Theorem 2.1. 
Secondly, we will use the following notion of $s w$-reducedness: The spectrum $\mathfrak{X}=\left(X_{k}, R_{j}^{k}\right)$ is called stepwise reduced (sw-reduced) if

$$
\forall j \exists L \geq j \forall J \geq j, \forall l \exists k: R_{L}^{j}\left(X_{L, l}\right) \subset R_{J}^{j}\left(X_{J, k}\right)+B_{j, k} .
$$

The author is indebted to P. Domański (Poznań) for suggesting this notion.

The statement in (1.8) implies that $\mathfrak{X}$ is reduced in the sense of Braun and Vogt $([9$, p. 150]), that is,

$$
\forall N \exists L \forall K \geq L \text { : the closure of } R_{K}^{N}\left(X_{K}\right) \text { in } X_{N} \text { contains } R_{L}^{N}\left(X_{L}\right) .
$$

The use of $s w$-reducedness will give Theorem 1.5 below and thus the implication $(a) \Rightarrow(b)$ in our Main Theorem 2.1. The statement in 2.1(b) is important for the transition to the hyperfunction criterion in part (e)(ii) of the Main Theorem 2.1.

We start with the following result translating the crucial points from Braun and Vogt [9] to our situation: that:

Lemma 1.3. Let $\mathfrak{X}=\left(X_{k}, R_{j}^{k}\right)$ be reduced and satisfy $\left(\mathrm{P}_{3}\right)$. Assume also

(a) $\mathfrak{X}$ satisfies the following condition $\left(\overline{\mathrm{P}_{3}}\right)$ :

$\forall \mu \exists k \forall K \exists n \forall m \forall \varepsilon>0 \exists N, S: R_{k}^{\mu}\left(B_{k, m}\right) \subset S R_{K}^{\mu}\left(B_{K, N}\right)+\varepsilon B_{\mu, n}$.

(b) Passing to a subsequence in $\mathfrak{X}$, for each fixed $d \in \mathbb{N}$ the following condition $\left(\overline{\mathrm{P}_{3}}\right)^{\prime \prime}$ holds:

$$
\forall \mu \exists n \forall m \forall \varepsilon>0 \exists N, S: R_{\mu}^{\mu-1}\left(B_{\mu, m}\right) \subset S R_{\mu+d}^{\mu-1}\left(B_{\mu+d, N}\right)+\varepsilon B_{\mu-1, n} .
$$

Then there is an increasing sequence $\widetilde{n}(k) \in \mathbb{N}$ such that for any $k$ and $m$ there are $\widetilde{N}(k, m) \in \mathbb{N}$ and $\widetilde{S}(k, m)>0$ such that

$$
R_{k}^{k-1}\left(B_{k, m}\right) \subset \widetilde{S}(k, m) R_{k+d}^{k-1}\left(B_{k+d, \widetilde{N}(k, m)}\right)+B_{k-1},
$$

where

$$
B_{j}:=\bigcap_{l=1}^{j}\left(R_{j}^{l}\right)^{-1}\left(B_{l, \widetilde{n}(l)}\right) .
$$

Proof. (a) This follows from the proofs of Braun and Vogt [9, Lemmata 9 and 5].

(b) Passing to a subsequence, we may assume that $\left(\overline{\mathrm{P}_{3}}\right)$ holds with $k=$ $\mu+1$. This is then applied for $K:=\mu+d$ to obtain $\left(\overline{\mathrm{P}_{3}}\right)^{\prime \prime}$.

Since (1.10) is crucial for the present paper, we include its proof here for the convenience of the reader, though it is mainly a notational variant of the corresponding proof of [9, Lemma 7]: We define $\widetilde{S}, \widetilde{N}$ and $\widetilde{n}$ inductively for $k \geq 2$, using $n(k), S(k, m, \varepsilon)$ and $N(k, m, \varepsilon)$ from $\left(\overline{\mathrm{P}_{3}}\right)^{\prime \prime}$ for $k$ instead of $\mu$ : 


$$
\begin{aligned}
& \widetilde{S}(k, m):= S\left(k, m, \varepsilon_{k}\right), \\
& \text { where } \varepsilon_{2}:=1 \text { and } \varepsilon_{k}:=1 /(2(1+\widetilde{S}(k-1, n(k)))) \text { for } k \geq 3, \\
& \widetilde{N}(2, m):=N(2, m, 1), \\
& \widetilde{N}(k, m):=\left\{\begin{array}{ll}
N\left(k, \widetilde{N}(k-1, n(k)), \varepsilon_{k}\right) & \text { if } m<\widetilde{N}(k-1, n(k)) \\
N\left(k, m, \varepsilon_{k}\right) & \text { if } m \geq \widetilde{N}(k-1, n(k))
\end{array} \text { for } k \geq 3,\right. \\
& \widetilde{n}(1):=n(2), \text { for } k \geq 3 .
\end{aligned}
$$

The claim for $k=2$ then follows from $\left(\overline{\mathrm{P}_{3}}\right)^{\prime \prime}$. Let $k \geq 3$ and assume that the claim holds for $k-1$. Let $u \in B_{k, m}$ be given. By $\left(\overline{\mathrm{P}_{3}}\right)^{\prime \prime}$ we can decompose $R_{k}^{k-1}(u)$ :

$$
R_{k}^{k-1}(u)=R_{k+d}^{k-1}\left(v_{k-1}\right)+u_{k-1}
$$

for some

$$
v_{k-1} \in S B_{k+d, N\left(k, m, \varepsilon_{k}\right)} \quad \text { and } \quad u_{k-1} \in \varepsilon_{k} B_{k-1, n(k)} .
$$

By the induction hypothesis for $m=n(k)$ we can decompose $R_{k-1}^{k-2}(u)$ :

$$
R_{k-1}^{k-2}\left(u_{k-1}\right)=R_{k-1+d}^{k-2}\left(v_{k-2}\right)+\widetilde{u}_{k-2}
$$

for some

$$
v_{k-2} \in \varepsilon_{k} \widetilde{S}(k-1, n(k)) B_{k-1+d, \widetilde{N}(k-1, n(k))} \subset \frac{1}{2} B_{k-1+d, \widetilde{N}(k-1, n(k))}
$$

(since $\left.\varepsilon_{k} \widetilde{S}(k-1, n(k)) \leq 1 / 2\right)$ and some

$$
\widetilde{u}_{k-2} \in \varepsilon_{k} B_{k-2} \text {. }
$$

Let

$$
u_{k-2}:=u_{k-1}-R_{k-1+d}^{k-1}\left(v_{k-2}\right)
$$

Then

$$
u_{k-2} \in \varepsilon_{k} B_{k-1, n(k)}+\varepsilon_{k} \widetilde{S}(k-1, n(k)) B_{k-1, \widetilde{N}(k-1, n(k))} \subset \frac{1}{2} B_{k-1, \widetilde{n}(k-1)}
$$

by $(1.12),(1.14)$ and the choice of $\widetilde{n}(k-1)$. Moreover,

$$
R_{k-1}^{k-2}\left(u_{k-2}\right)=R_{k-1}^{k-2}\left(u_{k-1}\right)-R_{k-1+d}^{k-2}\left(v_{k-2}\right)=\widetilde{u}_{k-2} \subset \frac{1}{2} B_{k-2}
$$

by (1.13) and (1.15), since $\varepsilon_{k} \leq 1 / 2$. Thus,

$$
u_{k-2} \in \frac{1}{2} B_{k-1} \text {. }
$$

By (1.11), (1.16), (1.12), (1.14) and (1.15) we get

$$
\begin{aligned}
R_{k}^{k-1}(u) & =R_{k-1+d}^{k-1}\left(v_{k-1}\right)+u_{k-1}=R_{k+d}^{k-1}\left(v_{k-1}\right)+R_{k-1+d}^{k-1}\left(v_{k-2}\right)+u_{k-2} \\
& \in S R_{k+d}^{k-1}\left(B_{k+d, N\left(k, m, \varepsilon_{k}\right)}\right)+\frac{1}{2} R_{k}^{k-1}\left(B_{k-1+d, \widetilde{N}(k-1, n(k)}\right)+\frac{1}{2} B_{k-1} \\
& \in S R_{k+d}^{k-1}\left(B_{k+d, N\left(k, m, \varepsilon_{k}\right)}\right)+\frac{1}{2} R_{k}^{k-1}\left(B_{k, m}\right)+\frac{1}{2} B_{k-1}
\end{aligned}
$$


if $m \geq \widetilde{N}(k-1, n(k))$. This defines a decomposition

$$
R_{k}^{k-1}(u)=R_{k+d}^{k-1}\left(x_{1}\right)+R_{k}^{k-1}\left(y_{1}\right)+w_{1}
$$

and by induction we can find

$$
x_{j+1} \in 2^{-j} S B_{k+d, N\left(k, m, \varepsilon_{k}\right)}, y_{j+1} \in 2^{-j-1} B_{k, m}, \quad w_{j+1} \in 2^{-j-1} B_{k-1}
$$
such that

$$
R_{k}^{k-1}\left(y_{j}\right)=R_{k+d}^{k-1}\left(x_{j+1}\right)+R_{k}^{k-1}\left(y_{j+1}\right)+w_{j+1} \quad \text { for } j \geq 1 .
$$

Since $X_{k+d, N\left(k, m, \varepsilon_{k}\right)}$ and $X_{k-1, \widetilde{n}(k-1)}$ are Banach spaces, the series

$$
v:=\sum_{j \geq 1} x_{j} \in 2 S B_{k+d, N\left(k, m, \varepsilon_{k}\right)} \quad \text { and } \quad w:=\sum_{j \geq 1} w_{j} \in B_{k-1, \widetilde{n}(k-1)}
$$

converge and we see as in [9], by (1.18), that $w \in B_{k-1}$. Then (1.19) implies that

$$
R_{k}^{k-1}(u)=R_{k+d}^{k-1}(v)+w \in 2 S B_{k+d, N\left(k, m, \varepsilon_{k}\right)}+B_{k-1} .
$$

This ends the induction step since we have $B_{k, m} \subset B_{k, \widetilde{N}(k-1, n(k))}$ if $m<$ $\widetilde{N}(k-1, n(k))$.

THEOREM 1.4. If $\mathfrak{X}$ is a reduced projective (DFS)-spectrum with $\left(\mathrm{P}_{3}\right)$, then $\operatorname{Proj}^{1}(\mathfrak{X})=0$.

Proof. Since $\mathfrak{X}$ is reduced and satisfies $\left(\mathrm{P}_{3}\right)$ we have

$$
\forall j \exists k \forall K \exists n: R_{k}^{j}\left(X_{k}\right) \subset R_{K}^{j}\left(X_{K}\right)+B_{j, n}
$$

(use the proof of $[9$, Lemma 5$]$ ). Passing to a subsequence, we may thus assume that $\mathfrak{X}$ satisfies

$$
\forall k \exists n(k): R_{k}^{k-1}\left(X_{k}\right) \subset R_{k+2}^{k-1}\left(X_{k+2}\right)+B_{k-1, n(k)}
$$

and also (by Lemma 1.3 for $d=3$ )

$$
\forall k, m: R_{k}^{k-1}\left(B_{k, m}\right) \subset R_{k+3}^{k-1}\left(X_{k+3}\right)+B_{k-1},
$$

where

$$
B_{j}:=\bigcap_{l=1}^{j}\left(R_{j}^{l}\right)^{-1}\left(B_{l, \widetilde{n}(l)}\right) .
$$

We now proceed as in the proof of [9, Theorem 8], using (1.20) and (1.21):

$$
R_{k}^{k-2}\left(X_{k}\right) \subset R_{k+2}^{k-2}\left(X_{k+2}\right)+R_{k-1}^{k-2}\left(B_{k-1, n(k)}\right) \subset R_{k+2}^{k-2}\left(X_{k+2}\right)+B_{k-2} .
$$

Passing to the subsequence with even indices, for any $k$ we thus get

$$
R_{k}^{k-1}\left(X_{k}\right) \subset R_{k+1}^{k-1}\left(X_{k+1}\right)+B_{k-1} .
$$

This implies that $\operatorname{Proj}^{1}(\mathfrak{X})=0$ (see the proof of Vogt [37, Theorem 4.4]).

We now consider the following situation: Let

$$
0 \rightarrow \mathfrak{X} \rightarrow \mathfrak{Y} \stackrel{\left(T_{k}\right)}{\longrightarrow} \mathfrak{Z} \rightarrow 0
$$


be an exact sequence of projective (DFS)-spectra $\mathfrak{X}=\left(X_{k}, R_{j}^{k}\right), \mathfrak{Y}=$ $\left(Y_{k}, R_{j}^{k}\right)$ and $\mathfrak{Z}=\left(Z_{k}, R_{j}^{k}\right)$. Let $X, Y$ and $Z$ be the projective limits of $\mathfrak{X}, \mathfrak{Y}$ and $\mathfrak{Z}$, respectively, and let $T: Y \rightarrow Z$ be the mapping defined by $\left(T_{k}\right)$.

ThEOREM 1.5. In the situation above, let $\mathfrak{X}$ be sw-reduced and satisfy $\left(\mathrm{P}_{3}\right)$. Then for any sequence $\mu(j) \in \mathbb{N}$ there is a sequence $\widetilde{\mu}(j) \in \mathbb{N}$ such that the following holds: If $g \in Z$ satisfies $g_{j}:=R^{j}(g) \in Z_{j, \mu(j)}$ for any $j$, then there is $f \in Y$ such that

$$
T(f)=g \quad \text { and } \quad R^{j}(f) \in Y_{j, \widetilde{\mu}(j)} \quad \text { for any } j .
$$

Proof. (a) When proving Theorem 1.5 we may pass to equivalent projective (DFS)-spectra $\widetilde{\mathfrak{X}}=\left(\widetilde{X}_{k}, \widetilde{R}_{j}^{k}\right), \widetilde{\mathfrak{Y}}=\left(\widetilde{Y}_{k}, \widetilde{R}_{j}^{k}\right)$ and $\widetilde{\mathfrak{Z}}=\left(\widetilde{Z}_{k}, \widetilde{R}_{j}^{k}\right)$ defining the projective limits $\widetilde{X}, \widetilde{Y}$ and $\widetilde{Z}$ isomorphic to $X, Y$ and $Z$, respectively.

(I) Suppose the statement is proved for the spectra $\widetilde{\mathfrak{X}}, \widetilde{\mathfrak{Y}}$ and $\widetilde{\mathfrak{Z}}$ and the mapping $\widetilde{T}: \widetilde{Y} \rightarrow \widetilde{Z}$ corresponding to $T$. Let

$$
\varphi:=\left(\varphi_{\lambda(j)}^{j}\right)_{j}: \widetilde{\mathfrak{Y}} \rightarrow \mathfrak{Y} \quad \text { and } \quad \Phi:=\left(\Phi_{\lambda(j)}^{j}\right)_{j}: \mathfrak{Y} \rightarrow \widetilde{\mathfrak{Y}}
$$

and

$$
\psi:=\left(\psi_{\kappa(j)}^{j}\right)_{j}: \widetilde{\mathfrak{Z}} \rightarrow \mathfrak{Z} \quad \text { and } \quad \Psi:=\left(\Psi_{\kappa(j)}^{j}\right)_{j}: \mathfrak{Z} \rightarrow \widetilde{\mathfrak{Z}}
$$

define the respective equivalence maps. Let $\mu(j)$ and $g \in Z$ be given such that $g_{j}:=R^{j}(g) \in Z_{j, \mu(j)}$ for any $j$. By Grothendieck's lemma (Meise and Vogt [30, 24.33]) there is $\nu(j)$ such that $\Psi_{\kappa(j)}^{j}\left(R^{\kappa(j)}(g)\right)=: \widetilde{g}_{j} \in \widetilde{Z}_{j, \nu(j)}$ for any $j$. Now, $\left(\widetilde{g}_{j}\right)_{j}$ defines $\widetilde{g} \in \widetilde{Z}$ and $\widetilde{R}^{j}(\widetilde{g})=\widetilde{g}_{j} \in \widetilde{Z}_{j, \nu(j)}$. Choose $\widetilde{\mu}(j)$ for $\nu(j)$ by the statement for $\widetilde{\mathfrak{X}}, \widetilde{\mathfrak{Y}}$ and $\widetilde{\mathfrak{Z}}$ and then $\widetilde{f} \in \widetilde{Y}$ such that $\widetilde{T}(\widetilde{f})=\widetilde{g}$ and $\widetilde{R}^{j}(\widetilde{f}) \in \widetilde{Y}_{j, \widetilde{\mu}(j)}$ for any $j$. Again by Grothendieck's lemma there is $\widetilde{\nu}(j)$ such that

$$
f_{j}:=\varphi_{\lambda(j)}^{j}\left(\widetilde{R}^{\lambda(j)}(\widetilde{f})\right) \in Y_{j, \widetilde{\nu}(j)} \quad \text { for any } j .
$$

Then $\left(f_{j}\right)_{j}$ defines $f \in Y, T(f)=g$ and $R^{j}(f)=f_{j} \in Y_{j, \widetilde{\nu}(j)}$. The claim thus holds for the spectra $\mathfrak{X}, \mathfrak{Y}$ and $\mathfrak{Z}$ with $\widetilde{\nu}(j)$ instead of $\widetilde{\mu}(j)$.

(II) Using the continuity of the equivalence maps and Grothendieck's lemma as in $(\mathrm{I})$, one proves that $\left(\mathrm{P}_{3}\right)$ and $s w$-reducedness are inherited from $\mathfrak{X}$ by the equivalent (DFS)-spectrum $\widetilde{\mathfrak{X}}$.

(b) Passing to equivalent spectra, we may assume that the following statements (i)-(iv) hold:

(i) $T_{j}: Y_{j} \rightarrow Z_{j}$ is continuous.

Since (1.22) is an exact sequence of spectra, we may assume that

$$
T_{j}\left(Y_{j}\right) \supset R_{j+1}^{j}\left(Z_{j+1}\right) .
$$

The mapping

$$
T_{j}^{-1} \circ R_{j+1}^{j}: Z_{j+1} \rightarrow Y_{j} / \operatorname{ker} T_{j}
$$


is continuous by the closed graph theorem for (DFS)-spaces. Since $Y_{j} / \operatorname{ker} T_{j}$ is isomorphic to $\operatorname{limind}_{k}\left(Y_{j, k} / \operatorname{ker}\left(\left.T_{j}\right|_{Y_{j, k}}\right)\right)$, Grothendieck's lemma implies that for any $k$ there is $\widehat{k}$ such that

$$
T_{j}^{-1} \circ R_{j+1}^{j}: Z_{j+1, k} \rightarrow Y_{j, \widehat{k}} / \operatorname{ker}\left(\left.T_{j}\right|_{Y_{j, \hat{k}}}\right) \text { is continuous. }
$$

Thus, we have

(ii) $\forall k \exists \widehat{k}: T_{j}\left(Y_{j, \widehat{k}}\right) \supset R_{j+1}^{j}\left(Z_{j+1, k}\right)$.

Since $(1.22)$ is exact at $\mathfrak{X}, \mathfrak{X}$ is equivalent to a spectrum of linear subspaces of $\left(Y_{j}\right)$. Since $(1.22)$ is exact at $\mathfrak{Y}, \mathfrak{X}$ is equivalent to the spectrum $\left(\operatorname{ker} T_{k}, R_{j}^{k}\right)$ (compare [38, remark before 1.3]). We may therefore assume that

(iii) $X_{j}=\operatorname{ker}\left(T_{j}\right)$ with the step spaces induced by $Y_{j}$.

Instead of the step spaces $X_{j, 1}, X_{j, 2}, \ldots$ for $X_{j}$ we can also take the step spaces $0, \ldots, 0, X_{j, 1}, X_{j, 2}, \ldots$ for $X_{j}$ (with $j$ times the null space in front, similarly for $Y_{j}$ ). Then passing to a subsequence (in the second index), we may also assume that for any $j \geq k$ and any $n$,

$$
R_{j}^{k}\left(X_{j, n}\right) \subset X_{k, n} \quad \text { and } \quad R_{j}^{k}\left(Y_{j, n}\right) \subset Y_{k, n}
$$

(see $[9,(1)]$ ). Passing to a subsequence (in the first index), we assume that $\mathfrak{X}$ satisfies $\left(\overline{\mathrm{P}_{3}}\right)^{\prime \prime}$ for $d=2$ and also (since $\mathfrak{X}$ is sw-reduced)

$$
\forall j, l \exists k: R_{j}^{j-1}\left(X_{j, l}\right) \subset R_{j+1}^{j-1}\left(X_{j+1, k}\right)+B_{j-1, k} .
$$

Now Lemma 1.3 gives

$$
\begin{aligned}
R_{k}^{k-1}\left(B_{k, m}\right) & \subset \widetilde{S}(k, m) R_{k+2}^{k-1}\left(B_{k+2, \widetilde{N}(k, m)}\right)+B_{k-1} \\
& \subset R_{k+2}^{k-1}\left(X_{k+2, \widetilde{N}(k, m)}\right)+B_{k-1},
\end{aligned}
$$

where

$$
B_{j}:=\bigcap_{l=1}^{j}\left(R_{j}^{l}\right)^{-1}\left(B_{l, \widetilde{n}(l)}\right) \subset B_{j, \widetilde{n}(j)} .
$$

(iv) We have

$$
\forall j, l \exists \widetilde{k}: R_{j}^{j-2}\left(X_{j, l}\right) \subset R_{j+1}^{j-2}\left(X_{j+1, \widetilde{k}}\right)+2^{-j} B_{j-2} .
$$

Indeed, fix $j$ and $l$ and choose $k$ by (1.24). Then (1.24) and (1.26) imply

$$
\begin{aligned}
R_{j}^{j-2}\left(X_{j, l}\right) & =R_{j-1}^{j-2}\left(R_{j}^{j-1}\left(X_{j, l}\right)\right) \subset R_{j-1}^{j-2}\left(R_{j+1}^{j-1}\left(X_{j+1, k}\right)+B_{j-1, k}\right) \\
& \subset R_{j+1}^{j-2}\left(X_{j+1, k}\right)+R_{j+1}^{j-2}\left(X_{j+1, \tilde{N}(j-1, k)}\right)+B_{j-2} \\
& \subset R_{j+1}^{j-2}\left(X_{j+1, \widetilde{k}}\right)+B_{j-2}
\end{aligned}
$$


for $\widetilde{k}:=\max (k, \tilde{N}(j-1, k))$. Since $X_{j, l}$ and $X_{j+1, \widetilde{k}}$ are linear spaces, this shows (1.27).

(c) Using the reductions and results from (b), we now start to prove the theorem: Let $\mu(j)$ and $g \in Z$ be as above. By (b)(ii) there are

$$
f_{j} \in Y_{j, \mu_{1}(j)} \text { such that } T_{j}\left(f_{j}\right)=R_{j+1}^{j}\left(g_{j+1}\right)=g_{j},
$$

where $\mu_{1}(j) \geq \mu(j+1)^{\wedge}$ can be chosen increasing and $g_{j}:=R_{j}(g)$. We now prove by induction that for $j \geq 3$ there are $\mu_{2}(j) \in \mathbb{N}$ with $\mu_{2}(j) \geq \mu_{1}(j+1)$ and $h_{j} \in X_{j, \mu_{2}(j)}$ such that

$$
R_{j+1}^{j-2}\left(f_{j+1}+h_{j+1}\right)-R_{j}^{j-2}\left(f_{j}+h_{j}\right) \in 2^{-j} B_{j-2} .
$$

Let $h_{3}:=0$ and $\mu_{2}(3):=\mu_{1}(4)$. If $h_{j}$ is chosen, we set $\widetilde{F}:=R_{j+1}^{j}\left(f_{j+1}\right)-$ $f_{j}-h_{j}$ and get $\widetilde{F} \in X_{j}$ by (b)(iii) and (1.28) since $h_{j} \in X_{j}$. Since $f_{j+1} \in$ $Y_{j+1, \mu_{1}(j+1)}$ and $h_{j} \in X_{j, \mu_{2}(j)}$ we have $\widetilde{F} \in X_{j, \mu_{2}(j)}$ by (1.23) since $\mu_{1}(j) \leq$ $\mu_{1}(j+1) \leq \mu_{2}(j)$. By $(1.27)$ we can thus find $\widetilde{k}=: \mu_{2}(j+1) \geq \mu_{1}(j+2)$ and $h_{j+1} \in X_{j+1, \mu_{2}(j+1)}$ such that

$$
R_{j}^{j-2}(\widetilde{F})+R_{j+1}^{j-2}\left(h_{j+1}\right) \in 2^{-j} B_{j-2} .
$$

This shows (1.29).

(d) For $j \geq k \geq 2$ we have

$$
\begin{aligned}
& R_{j}^{k}\left(f_{j}+h_{j}\right) \\
& \quad=R_{k+2}^{k}\left(f_{k+2}+h_{k+2}\right)+\sum_{l=k+2}^{j-1} R_{l-2}^{k}\left(R_{l+1}^{l-2}\left(f_{l+1}+h_{l+1}\right)-R_{l}^{l-2}\left(f_{l}+h_{l}\right)\right) \\
& \quad \in Y_{k, \mu_{2}(k+2)}+\sum_{l=k+2}^{j-1} 2^{-l} R_{l-2}^{k}\left(B_{l-2}\right) \subset Y_{k, \mu_{2}(k+2)}+\left(\sum_{l=k+2}^{j-1} 2^{-l}\right) B_{k} \\
& \quad \subset Y_{k, \mu_{2}(k+2)}+\left(\sum_{l=k+2}^{j-1} 2^{-l}\right) B_{k, \nu(k)}
\end{aligned}
$$

by (1.23) and (1.26). Hence,

$$
F_{k}:=\lim _{j \rightarrow \infty} R_{j}^{k}\left(f_{j}+h_{j}\right) \text { exists in } Y_{k, \widetilde{\mu}(k)} \text { for any } k,
$$

where $\widetilde{\mu}(k):=\max \left(\mu_{2}(k+2), \nu(k)\right)$. For $l \leq k$ we have

$$
R_{k}^{l}\left(F_{k}\right)=\lim _{j \rightarrow \infty} R_{k}^{l}\left(R_{j}^{k}\left(f_{j}+h_{j}\right)\right)=\lim _{j \rightarrow \infty} R_{j}^{l}\left(f_{j}+h_{j}\right)=F_{l} .
$$


Thus, $\left(F_{k}\right)_{k}$ defines $F \in \operatorname{limproj}_{k} Y_{k}=Y$ with $R^{k}(F)=F_{k} \in Y_{k, \widetilde{\mu}(k)}$ for any $k$. We have $T(F)=g$ since $h_{j} \in X_{j}=\operatorname{Ker}\left(T_{j}\right)$ and therefore

$$
\begin{aligned}
T_{k}\left(F_{k}\right) & =\lim _{j \rightarrow \infty} T_{k}\left(R_{j}^{k}\left(f_{j}+h_{j}\right)\right) \\
& =\lim _{j \rightarrow \infty} R_{j}^{k}\left(T_{j}\left(f_{j}\right)\right)=\lim _{j \rightarrow \infty} R_{j}^{k}\left(R_{j+1}^{j}\left(g_{j+1}\right)\right)=g_{k}
\end{aligned}
$$

by (1.28).

Notice that condition $\left(\mathrm{P}_{3}\right)$ is weaker than Vogt's condition $\left(\mathrm{P}_{2}\right)$ from [37]. The latter condition follows from the surjectivity of $T: Y \rightarrow Z$ if $\mathfrak{X}, \mathfrak{Y}$ and $\mathfrak{Z}$ are projective (DFS)-spectra and if $\operatorname{Proj}^{1}(\mathfrak{Y})=0$ (use Vogt [37, Theorems 5.2 and 2.7]). Assuming that $\mathfrak{X}$ is $s w$-reduced, Theorem 1.5 thus provides an automatic improvement on the solutions in this situation if the surjectivity of $T$ is known.

We will use Theorem 1.5 in Section 4 to prove that the weak technical condition $\left(\underline{\mathrm{A}_{\Omega}}\right)$ is sufficient for the surjectivity of $P(D)$ on $A(\Omega)$. For this we have to check first that the sequence (1.3) of projective spectra is exact. Several equivalent criteria for this are given in Proposition 3.3, including the condition $\left(\mathrm{C}_{\Omega}\right)$ which obviously follows from $\left(\underline{\mathrm{A}_{\Omega}}\right)$. Next we have to show that the kernel spectrum $N_{P}^{\Omega}$ is $s w$-reduced (see Proposition 4.3, which is based on Lemma 4.2). The main point however is to prove condition $\left(\mathrm{P}_{3}\right)$ for $N_{P}^{\Omega}$, which means that we have to prove a decomposition with bounds in $N_{P}^{\Omega}$. This decomposition is obtained in Theorem 4.5, essentially by using a cutting off procedure, which of course leads out of $N_{P}^{\Omega}$, and then repairing this defect by means of the existence result for $P(D)$ from Theorem 4.4, which is the analytic core of this part of our results.

2. The characterization. In this section we will state the new characterization of surjective partial differential operators on $A(\Omega)$ and prove some direct applications. The proof of the Main Theorem 2.1 is postponed to the subsequent sections. To state the theorem, we first introduce some notation: Let

$$
G(x, y):=-|(x, y)|^{1-n} /\left((n-1) c_{n+1}\right)
$$

be the canonical even elementary solution of $\Delta$ (see Hörmander [14, Theorem 3.3.2], and recall that $n+1 \geq 3)$. For $T \in \mathbb{R}$ let

$$
\begin{aligned}
\mathfrak{U}(\Omega \times\{T\}) & :=\left\{V \subset \mathbb{R}^{n+1} \mid V \text { is open and } V \cap\left(\mathbb{R}^{n} \times\{T\}\right)=\Omega \times\{T\}\right\}, \\
\mathfrak{U}(\Omega) & :=\mathfrak{U}(\Omega \times\{0\}) .
\end{aligned}
$$

For $\delta>0$ let

$$
A_{\delta}(\omega):=\left\{f \in A(\omega)\left|\sup _{x \in \omega}\right| f^{(a)}(x) \mid \delta^{|a|} / a !<\infty\right\} .
$$


The hyperfunctions on $\Omega$ can be defined by

$$
\mathfrak{B}(\Omega):=\widetilde{C}_{\Delta}(\Omega \times(\mathbb{R} \backslash\{0\})) / \widetilde{C}_{\Delta}(\Omega \times \mathbb{R})
$$

(see Bengel [4] and Hörmander [14, Chapter IX]). Here $\widetilde{C}_{\Delta}(V)$ is the space of harmonic functions on $V$ which are even with respect to $y$.

Our characterization of surjective partial differential operators on $A(\Omega)$ is contained in the following Main Theorem of this paper:

THEOREM 2.1. Let $\Omega \subset \mathbb{R}^{n}$ be open. The following statements are equivalent:

(a) $P(D): A(\Omega) \rightarrow A(\Omega)$ is surjective.

(b) For any $V \in \mathfrak{U}(\Omega)$ there is $W \in \mathfrak{U}(\Omega)$ with $W \subset V$ such that

$$
\left.P(D) C_{\Delta}(W) \supset C_{\Delta}(V)\right|_{W} .
$$

(c) $P(D)$ satisfies the following condition $\left(\mathrm{A}_{\Omega}\right)$ : for any $\omega \subset \subset \Omega$ there is $\widetilde{\omega} \subset \subset \Omega$ with $\widetilde{\omega} \supset \supset \omega$ such that for any $\widehat{\omega} \subset \subset \bar{\subset}$ and any $\xi \in \partial \widetilde{\omega}$ there is $\delta>0$ such that for any $0<T \leq \delta$ there are $V \in \mathfrak{U}(\widehat{\omega} \times\{T\})$ and $E \in C_{\Delta}(X)$ such that

$$
P(D) E=G(\cdot-\xi, \cdot) \quad \text { on } X:=V \cup(\omega \times] T-\delta, T+\delta[) .
$$

(d) $P(D)$ satisfies the following condition $\left(\overline{\mathrm{A}_{\Omega}}\right)$ : for any $\omega \subset \subset \Omega$ there are $\widetilde{\omega} \subset \subset \Omega$ and $\delta>0$ such that for any $\xi \in \Omega \backslash \widetilde{\omega}$ and any $0<T \leq 1$ there are $V \in \mathfrak{U}(\Omega \times\{T\})$ and $E \in C_{\Delta}(X)$ such that (2.2) holds.

(e) (i) For any $g \in A(\Omega)$ and any $\omega \subset \subset \Omega$ there is $f \in A(\omega)$ such that

$$
P(D) f=\left.g\right|_{\omega} .
$$

(ii) $P(D)$ satisfies the following condition $\left(\mathrm{B}_{\Omega}\right)$ : for any $\omega \subset \subset \Omega$ there is $\widetilde{\omega} \subset \subset \Omega$ with $\widetilde{\omega} \supset \supset \omega$ such that for any $\xi \in \partial \widetilde{\omega}$ and any $\widehat{\omega} \subset \subset \Omega$ there is $F \in \mathfrak{B}(\widehat{\omega})$ such that

$$
P(D) F=\delta_{\xi} \quad \text { on } \widehat{\omega} \quad \text { and }\left.\quad F\right|_{\omega} \in A(\omega),
$$

where $\delta_{\xi}$ is the point evaluation at $\xi$.

(f) $P(D)$ satisfies $(\mathrm{e})(\mathrm{i})$ and the following condition $\left(\overline{\mathrm{B}_{\Omega}}\right)$ : for any $\omega \subset \subset$ $\Omega$ there are $\widetilde{\omega} \subset \subset \Omega$ and $\delta>0$ such that for any $\xi \in \Omega \backslash \widetilde{\omega}$ there is $F \in \mathfrak{B}(\Omega)$ such that

$$
P(D) F=\delta_{\xi} \quad \text { on } \Omega \quad \text { and }\left.\quad F\right|_{\omega} \in A_{\delta}(\omega)
$$

(g) $P(D)$ satisfies (e)(i) and the following condition: for any $\omega \subset \subset \Omega$ there are $\widetilde{\omega} \subset \subset \Omega$ with $\widetilde{\omega} \supset \omega$ and $\delta>0$ such that for any $0<\widehat{\delta} \leq \delta$ there is $\gamma>0$ such that for any $H \in \mathfrak{B}(\Omega)$ with $\left.H\right|_{\widetilde{\omega}} \in A_{\widehat{\delta}}(\widetilde{\omega})$ there is $F \in \mathfrak{B}(\Omega)$ such that

$$
P(D) F=H \quad \text { on } \Omega \quad \text { and }\left.\quad F\right|_{\omega} \in A_{\gamma}(\omega) .
$$


Condition $\left(\overline{\mathrm{B}_{\Omega}}\right)$ is similar to a condition which is equivalent to the surjectivity of $P(D)$ on nonquasianalytic classes of ultradifferentiable functions of Roumieu type (Langenbruch [22, 25, 23]).

The canonical elementary solution $G$ of $\Delta$ is used in (2.2) since $G$ provides the basic isomorphism of $C_{\Delta}(K)^{\prime}$ and $C_{\Delta, 0}\left(\mathbb{R}^{n+1} \backslash K\right)$ in the Grothendieck-Tillmann duality (see (3.3)).

Condition $\left(\overline{\mathrm{B}_{\Omega}}\right)$ is an improvement of the necessary condition from Langenbruch [24, Theorem 1.3], where a weaker notion of (shifted) elementary solution was used.

The condition in Theorem $2.1(\mathrm{~g})$ roughly means that the equation $P(D) F=H$ may be solved in $\mathfrak{B}(\Omega)$ respecting lacunas in the analytic singular support. This corresponds to the characterization of surjectivity of $P(D)$ in nonquasianalytic Gevrey classes (see Langenbruch [22, Theorem $2.1(\mathrm{c})])$.

We already used the necessary condition from Langenbruch [24, Theorem 1.3] to show that local hyperbolicity of the principal part $P_{m}$ of $P$ and hyperbolicity of the localizations of $P_{m}$ at $\infty$ are necessary for the surjectivity of $P(D)$ on $A(\Omega)$ in many situations (see Langenbruch [24, 26]).

In the following discussion of the Main Theorem we will therefore concentrate on obtaining weak forms of sufficient (or equivalent) conditions for surjectivity and relate these to the corresponding results in the literature.

As a first consequence, we notice that condition (e)(i) is redundant if the shifted elementary solutions $F$ are defined on $\operatorname{conv}(\widehat{\omega})$ :

COROLlary 2.2. $P(D)$ is surjective on $A(\Omega)$ if for any $\omega \subset \subset \Omega$ there is $\widetilde{\omega} \subset \subset \Omega$ with $\widetilde{\omega} \supset \supset \omega$ such that for any $\widehat{\omega} \subset \subset \Omega$ and any $\xi \in \partial \widetilde{\omega}$ there is $F \in \mathfrak{B}(\operatorname{conv}(\widehat{\omega}))$ such that

$$
P(D) F=\delta_{\xi} \quad \text { on } \operatorname{conv}(\widehat{\omega}) \quad \text { and }\left.\quad F\right|_{\omega} \in A(\omega) .
$$

Proof. We show $\left(\underline{\mathrm{A}_{\Omega}}\right)$ : Let $\omega \subset \subset \omega_{1} \subset \subset \Omega$. Choose $\widetilde{\omega}$ for $\omega_{1}$ by the assumption. For $\xi \in \overline{\partial \widetilde{\omega}}$, choose $F \in \mathfrak{B}\left(\widehat{\omega}_{1}\right)$ for $\widehat{\omega}_{1}:=\operatorname{conv}(\widehat{\omega})$ by the assumption. By the definition (2.1) of hyperfunctions, $F$ is defined by some $F_{1} \in \widetilde{C}_{\Delta}\left(\widehat{\omega}_{1} \times(\mathbb{R} \backslash\{0\})\right)$ such that for some $H \in D^{\prime}\left(\widehat{\omega}_{1} \times \mathbb{R}\right)$ we have

$$
P(D) F_{1}=\left.H\right|_{\widehat{\omega}_{1} \times(\mathbb{R} \backslash\{0\})} \quad \text { and } \quad \Delta H=\delta_{(\xi, 0)} \quad \text { on } \widehat{\omega}_{1} \times \mathbb{R} .
$$

Moreover, $F_{2}:=\left.F_{1}\right|_{\left.\widehat{\omega}_{1} \times\right] 0, \infty[}$ can be extended as a harmonic function to $V \cup\left(\widehat{\omega}_{1} \times\right] 0, \infty[)$ for some neighbourhood $V \in \mathfrak{U}\left(\omega_{1}\right)$. Hence there is $\delta>0$ such that $F_{2}$ can be uniquely extended to a harmonic function $F_{3}$ defined on

$$
W:=(\omega \times]-\delta, \infty[) \cup\left(\widehat{\omega}_{1} \times\right] 0, \infty[) .
$$

By (2.4) there is $h \in C_{\Delta}\left(\widehat{\omega}_{1} \times \mathbb{R}\right)$ such that $H=G(\cdot-\xi, \cdot)+h$. Since $\Delta h=0$ 
and $\widehat{\omega}_{1} \times \mathbb{R}$ is convex, we may solve the overdetermined system

$$
\left\{\begin{array}{l}
P(D) g=h \\
\Delta g=0
\end{array} \text { on } \widehat{\omega}_{1} \times \mathbb{R}\right.
$$

by the fundamental principle of Ehrenpreis/Palamodov. $E:=F_{3}-\left.g\right|_{W}$ then satisfies

$$
P(D) E=G(\cdot-\xi, \cdot) \quad \text { on } W
$$

by harmonic continuation. Since

$$
\omega \times] T-\delta / 2, T+\delta / 2[\subset W \in \mathfrak{U}(\widehat{\omega} \times\{T\})
$$

for $0<T \leq \delta / 2$, this shows $\left(\underline{\mathrm{A}_{\Omega}}\right)$.

Corollary 2.3. Let $\Omega$ be convex. Then $P(D)$ is surjective on $A(\Omega)$ iff $P(D)$ satisfies $\left(\underline{\mathrm{B}_{\Omega}}\right)$ iff $P(D)$ satisfies $\left(\overline{\mathrm{B}_{\Omega}}\right)$.

Proof. The necessity follows from Theorem 2.1, the sufficiency follows from Corollary 2.3 since $\widehat{\omega}$ can be chosen convex.

The two main examples of convex open sets are $\mathbb{R}^{n}$ and a halfspace. We state the respective conditions for these cases explicitly. Let

$$
U_{\varepsilon}(\xi):=\left\{x \in \mathbb{R}^{n}|| x-\xi \mid<\varepsilon\right\} \quad \text { and } \quad U_{\varepsilon}:=U_{\varepsilon}(0) .
$$

EXAmple 2.4. $P(D)$ is surjective on $A\left(\mathbb{R}^{n}\right)$ if and only if for any $j \in \mathbb{N}$ there is $k \in \mathbb{N}$ such that for any $\xi \in \mathbb{R}^{n}$ with $|\xi| \geq k$ there is an elementary solution $F \in \mathfrak{B}\left(\mathbb{R}^{n}\right)$ of $P(D)$ such that $F$ is real analytic on $U_{j}(\xi)$.

Proof. The stated condition directly follows from $\left(\overline{\mathrm{B}_{\mathbb{R}^{n}}}\right)$ and it implies $\left(\underline{\mathrm{B}_{\mathbb{R}^{n}}}\right)$. So the claim is a consequence of Corollary 2.3 .

In the discussion of surjectivity of $P(D)$ on $A(\Omega)$, operators with locally hyperbolic principal part $P_{m}$ have been studied extensively. These are defined as follows: Let

$$
S^{n-1}:=\left\{x \in \mathbb{R}^{n}|| x \mid=1\right\} .
$$

Definition 2.5. $P_{m}$ is locally hyperbolic if for any $\Theta \in S^{n-1}$ there are $N \in S^{n-1}$ and $\delta>0$ such that for $(x, z) \in \mathbb{R}^{n} \times \mathbb{C}$,

$$
P_{m}(\Theta+x+z N) \neq 0 \quad \text { if }|(x, z)|<\delta \text { and } \operatorname{Im} z \neq 0 .
$$

If $P_{m}$ is locally hyperbolic at $\Theta$ with respect to $N$, then the localization $\left(P_{m}\right)_{\Theta}$ of $P_{m}$ at $\Theta$ is hyperbolic with respect to $N$. Recall that $\left(P_{m}\right)_{\Theta}$ is defined by

$$
\left(P_{m}\right)_{\Theta}:=\lim _{s \rightarrow 0}\left(P_{m}(\theta+s x) s^{-q_{\theta}}\right)
$$

where $s^{q_{\theta}}$ is the term of lowest order in the expansion of $\left(P_{m}\right)_{\Theta}$.

Let $\Gamma\left(\left(P_{m}\right)_{\Theta}, N\right)$ be the component of $N$ in $\left\{x \in \mathbb{R}^{n} \mid\left(P_{m}\right)_{\Theta}(x) \neq 0\right\}$. Then the dual cone of $\Gamma\left(\left(P_{m}\right)_{\Theta}, N\right)$ is called the local propagation cone for $P_{m}$ at $\Theta$ and is denoted by $K\left(\left(P_{m}\right)_{\Theta}, N\right)$. Obviously, $P_{m}$ is also locally 
hyperbolic with respect to $N$ for any $\widetilde{\Theta} \in S^{n-1}$ near $\Theta$. Moreover, the mapping $\Theta \mapsto K\left(\left(P_{m}\right)_{\Theta}, N\right)$ is locally upper semicontinuous, that is, for any $\varepsilon>0$ there is $\delta>0$ such that

$$
S^{n-1} \cap K\left(\left(P_{m}\right)_{\widetilde{\Theta}}, N\right) \subset S^{n-1} \cap\left(K\left(\left(P_{m}\right)_{\Theta}, N\right)+U_{\varepsilon}\right) \quad \text { if }|\Theta-\widetilde{\Theta}|<\delta .
$$

This means that $K\left(\left(P_{m}\right)_{\widetilde{\Theta}}, N\right)$ is contained in a conic $\varepsilon$-neighbourhood of $K\left(\left(P_{m}\right)_{\Theta}, N\right)$ if $|\Theta-\widetilde{\Theta}|<\delta$. The following basic result is due to Andersson [1, Corollary 4.1] (use also the remark of Andersson [2, p. 2] and (2.6)).

THEOREM 2.6. Let $P_{m}$ be locally hyperbolic at any $\Theta \in S^{n-1}$ with respect to $N_{\Theta} \in S^{n-1}$. Then for any $\varepsilon>0$ there are $\Theta_{1}, \ldots, \Theta_{k} \in S^{n-1}$ and a fundamental solution $F \in \mathfrak{B}\left(\mathbb{R}^{n}\right)$ for $P(D)$ which is analytic outside a conic $\varepsilon$-neighbourhood of

$$
\bigcup_{j \leq k} K\left(\left(P_{m}\right)_{\Theta_{j}}, N_{\Theta_{j}}\right) .
$$

Thus, if $P_{m}$ is locally hyperbolic, for any $x \in S^{n-1}$ there is an elementary solution $F \in \mathfrak{B}\left(\mathbb{R}^{n}\right)$ for $P(D)$ such that

$F$ is real analytic in a conic $\varepsilon$-neighbourhood $\Gamma$ of $x$.

Therefore, the criterion of Example 2.4 is satisfied if $P_{m}$ is locally hyperbolic, and $P(D)$ is thus surjective on $A\left(\mathbb{R}^{n}\right)$. This is the result of Andersson [2] who used (2.7) and some additional growth condition for $E$ to get the result by a decomposition of $A\left(\mathbb{R}^{n}\right)$ into a finite sum of real analytic functions decaying sufficiently fast. In some sense, Example 2.4 thus turns the part (2.7) of the sufficient criterion of Andersson into a characterization. Notice that for $n \geq 4$ there are operators which are surjective on $A\left(\mathbb{R}^{n}\right)$ though $P_{m}$ is not locally hyperbolic (Hörmander [13]).

We now discuss the case of halfspaces: For $\left.\left.0 \neq \eta \in \mathbb{R}^{n}, k \in\right] 0, \infty\right]$ and $\varepsilon \in \mathbb{R}$ let

$$
H(\eta)_{\varepsilon, k}:=\left\{x \in U_{k} \mid\langle x, \eta\rangle>\varepsilon\right\},
$$

where $H(\eta):=H(\eta)_{0, \infty}$ is the halfspace defined by $\eta$.

ExAmPle 2.7. $P(D)$ is surjective on $A(H(\eta))$ if and only if $P(D)$ satisfies $\left(\overline{\mathrm{B}_{\mathbb{R}^{n}}}\right)$ (or $\left(\mathrm{B}_{\mathbb{R}^{n}}\right)$ ) and for any $\varepsilon>0$ there is $\delta<0$ such that for any $k \in \mathbb{N}$ there is an elementary solution $F \in \mathfrak{B}\left(H(\eta)_{\delta, k}\right)$ of $P(D)$ such that $F$ is real analytic on $H(\eta)_{\varepsilon, 1 / \varepsilon}$.

Proof. " $\Rightarrow " P(D)$ is surjective on $A\left(\mathbb{R}^{n}\right)$ by Hörmander $[13]$, hence $\left(\overline{\mathrm{B}_{\mathbb{R}^{n}}}\right)$ holds by Theorem 2.1, and Theorem 2.1 also implies $\left(\mathrm{B}_{H(\eta)}\right)$. The second condition now follows from $\left(\mathrm{B}_{H(\eta)}\right)$.

" $\Leftarrow "$ By Corollary 2.3 we have to show $\left(\mathrm{B}_{H(\eta)}\right)$. Let $\omega \subset \subset H(\eta)$. By $\left(\underline{\mathrm{B}_{\mathbb{R}^{n}}}\right)$ there is $C>0$ such that for any $\xi \in \overline{\partial U_{C}}$ and any $k \in \mathbb{N}$ there is 
$F \in \mathfrak{B}\left(U_{k}\right)$ such that

$$
P(D) F=\delta_{\xi} \quad \text { on } U_{k} \quad \text { and }\left.\quad F\right|_{\omega} \in A(\omega) .
$$

Choose $0<\varepsilon \leq 1$ such that

$$
H_{2 \varepsilon, 1 / \varepsilon} \supset \supset \omega+\left\{x \in \bar{U}_{C} \mid\langle x, \eta\rangle=0\right\}=: \omega+\widetilde{U}_{C}
$$

and then choose $0<\delta<\varepsilon$ and $\widetilde{F} \in \mathfrak{B}\left(H(\eta)_{-\delta, k}\right)$ for $k \in \mathbb{N}$ by the assumption. For $x \in \widetilde{U}_{C}$ we set $\xi:=\delta \eta+x$ and $F_{\xi}:=\widetilde{F}(\cdot-\xi)$. Then $F_{\xi}$ is defined on $H(\eta)_{0, k-C}$,

$$
P(D) F_{\xi}=\delta_{\xi} \quad \text { on } H_{0, k-C} \quad \text { and }\left.\quad F\right|_{\omega} \in A(\omega)
$$

by $(2.8)$. This proves $\left(\mathrm{B}_{H(\eta)}\right)$ for $\widetilde{\omega}:=H(\eta)_{\delta, C}$.

Condition $\left(\mathrm{B}_{\mathbb{R}^{n}}\right)$ cannot be omitted in Example 2.7, since it does not follow from the second condition. Indeed, the canonical elementary solution of the heat operator $P(D):=\sum_{j \leq n-1} \partial_{j}^{2}-\partial_{n}$ vanishes on $H\left(e_{n}\right)$, so the second condition in Example 2.7 is clearly satisfied, but $P(D)$ is not surjective on $A\left(\mathbb{R}^{n}\right)$ for $n \geq 3$.

We now come to a sufficient criterion in the spirit of the results of Kawai [18], Kaneko [15-17] and Zampieri [42], involving only a condition for the boundary points of $\Omega$.

THEOREM 2.8. $P(D)$ is surjective on $A(\Omega)$ if $P(D)$ satisfies $\left(\underline{\mathrm{B}_{\mathbb{R}^{n}}}\right)$ and if for any $\zeta \in \partial \Omega$ and any $\omega \subset \subset \Omega$ there are a convex neighbourhood $U$ of $\bar{\Omega}$ and $F_{\zeta} \in \mathfrak{B}(U)$ such that

$$
P(D) F_{\zeta}=\delta_{\zeta} \quad \text { on } U \quad \text { and }\left.\quad F_{\zeta}\right|_{\omega} \in A(\omega) .
$$

If $\Omega$ is bounded, condition $\left(\mathrm{B}_{\mathbb{R}^{n}}\right)$ can be omitted.

Proof. We check the condition of Corollary 2.2. Fix $\omega \subset \subset \Omega$. By Theorem 2.1, $P(D)$ satisfies $\left(\overline{\mathrm{B}_{\mathbb{R}^{n}}}\right)$, hence there is $C>0$ such that for any $\xi \in \partial U_{C}$ there is $F \in \mathfrak{B}\left(\mathbb{R}^{n}\right)$ such that $P(D) F=\delta_{\xi}$ and $\left.F\right|_{\omega} \in A(\omega)$. Therefore, the condition of Corollary 2.2 must be checked only for those points $\xi \in \Omega \cap \bar{U}_{C}$ which are near the boundary. But for these, suitable hyperfunctions are provided by small shifts of $F_{\zeta}$ for $\zeta \in \partial \Omega$ from the assumption.

Corollary 2.9. $P(D)$ is surjective on $A(\Omega)$ if for any $\zeta \in \partial \Omega$ and any $\Theta \in S^{n-1}$ there is $N_{\Theta, \zeta} \in S^{n-1}$ such that $P_{m}$ is locally hyperbolic at $\Theta$ with respect to $N_{\Theta, \zeta}$ and

$$
\left(\zeta+K\left(\left(P_{m}\right)_{\Theta}, N_{\Theta, \zeta}\right)\right) \cap \Omega=\emptyset .
$$

Proof. Since $P_{m}$ is locally hyperbolic, $P(D)$ satisfies $\left(\mathrm{B}_{\mathbb{R}^{n}}\right)$ by $(2.7)$. Fix $\omega \subset \subset \Omega$ and $\zeta \in \partial \omega$. By (2.9) we may choose a conic neighbourhood $\Gamma$ of $\bigcup_{\Theta \in S^{n-1}} K\left(\left(P_{m}\right)_{\Theta}, N_{\Theta, \zeta}\right)$ such that

$$
(\zeta+\Gamma) \cap \omega=\emptyset .
$$


By Theorem 2.6 there is an elementary solution $\widetilde{F} \in \mathfrak{B}\left(\mathbb{R}^{n}\right)$ for $P(D)$ such that $\widetilde{F}$ is real analytic on $\mathbb{R}^{n} \backslash \Gamma$, hence $F:=\widetilde{F}(\cdot-\zeta)$ is real analytic on $\omega$ by (2.10). Thus $P(D) A(\Omega)=A(\Omega)$ by Theorem 2.8 .

The first results in the direction of Corollary 2.9 are due to Kawai [18]. For bounded $\Omega$, the result is due to Kaneko [16] (see also Kaneko [17]) using the approach of Kawai.

For convex $\Omega$, Corollary 2.9 was proved by Zampieri [42] using Hörmander's approach. For nonconvex $\Omega$, a different proof was given by Zampieri [43, Theorem 2.1] in the special case where $P_{m}$ decomposes into an elliptic and a hyperbolic factor.

The case of unbounded $\Omega$ was considered by Kaneko [15] using Fourier hyperfunctions. Additionally to the pointwise condition (2.9) he needed certain extra assumptions including boundary points at $\infty$, i.e. points in the closure of $\partial \Omega$ in the directional compactification $D^{n}$ of $\mathbb{R}^{n}$ (see Kaneko [16, Section 3]). These assumptions are redundant, since we only need shifted elementary solutions which are real analytic on $\omega \subset \subset \Omega$ (and not on $\Omega$ ), so (2.6) and Theorem 2.6 are sufficient in our approach.

As an easy example we now consider operators of real principal type. Recall that $P(D)$ is an operator of principal type if $P_{m}$ is real and if $\operatorname{grad} P_{m}(\theta)$ $\neq 0$ whenever $0 \neq \theta \in \mathbb{R}^{n}$ and $P_{m}(\theta)=0$. The set of bicharacteristic directions is then defined by

$\mathrm{BD}(P):=\left\{\xi \in \mathbb{R}^{n} \mid \xi=\operatorname{grad} P_{m}(\theta)\right.$ for some $0 \neq \theta \in \mathbb{R}^{n}$ with $\left.P_{m}(\theta)=0\right\}$.

Notice that operators of real principal type are locally hyperbolic with local propagation cones

$$
K\left(\left(P_{m}\right)_{\theta}, \operatorname{grad} P_{m}(\theta)\right)= \pm\left[0, \infty\left[\operatorname{grad} P_{m}(\theta) .\right.\right.
$$

$b_{(x, \xi)}:=x+\xi \mathbb{R}$ is called a bicharacteristic line if $x \in \mathbb{R}^{n}$ and $\xi \in \operatorname{BD}(P)$.

EXAMPLE 2.10. Let $P(D)$ be an operator of real principal type. Then $P(D)$ is surjective on $A(\Omega)$ if any bicharacteristic line $b_{(x, \xi)}$ issuing from $x \in \partial \Omega$ intersects $\Omega$ in an interval.

Proof. The statement is obvious from Corollary 2.9 and the remarks above.

The example improves on the corresponding results of Kawai [18, Theorem 3.1] and Kaneko [15, 16]. The example includes many classical operators, e.g. the wave equation $P(D):=\partial_{n}^{2}-\sum_{j=1}^{n-1} \partial_{j}^{2}$, the ultrahyperbolic operator $P(D):=\partial_{1}^{2}+\partial_{2}^{2}-\partial_{3}^{2}-\partial_{4}^{2}$ (for $n=4$ ) and Zeylon's operator $P(D)=\sum_{j=1}^{n} \partial_{j}^{3}$.

In fact, our results improve on those of Kaneko [15] already in the case of two variables, where we obtain the following characterization of Zampieri $[41,43]$ (for bounded open sets see Kawai [18, Theorem 5.7]): 
Corollary 2.11. The following are equivalent for an open set $\Omega \subset \mathbb{R}^{2}$ :

(a) $P(D)$ is surjective on $A(\Omega)$.

(b) Every characteristic line intersects $\Omega$ in an interval.

Proof. $(\mathrm{a}) \Rightarrow(\mathrm{b})$. Since $P(D)$ is surjective on $A(\Omega), \Omega$ is $P$-convex for supports by Zampieri [40]. Hence, (b) follows from Hörmander [14, Theorem 10.8.3].

(b) $\Rightarrow(\mathrm{a})$. Since $n=2$, any $P_{m}$ is locally hyperbolic, and the notions of a characteristic line and of the union of the positive and negative propagation cones coincide. Hence, the assumptions of Corollary 2.9 are satisfied by (b), and $P(D)$ is surjective on $A(\Omega)$ by that corollary.

In a remark on p. 345 of [15], Kaneko noticed that the results of that paper are not sufficient to show that the operator $P(D)=D_{1}$ is surjective on $A(\Omega)$ for $\Omega:=\left\{x \in \mathbb{R}^{2} \mid x_{1}<-x_{2}^{2}\right\}$, while this result clearly follows from Corollary 2.11 (or integration).

At the end of his paper [16], Kaneko conjectured that condition (2.9) was also necessary for surjectivity of $P(D)$ on $A(\Omega)$ if $\Omega$ is bounded. The following example shows that this is not true:

ExAmple 2.12. For $t \in \mathbb{R}$ let $\gamma(t):=(\cos (t), \sin (t))$. Let $\widetilde{\Omega}$ be the spiral in $\mathbb{R}^{3}$ defined by the disjoint (!) union

$$
\widetilde{\Omega}:=\bigcup_{t \in \mathbb{R}}(\gamma(t)] 1 / 2,2[\times] t-1, t+1[)
$$

and let

$$
\Omega:=\left\{x \in \widetilde{\Omega} \mid 0<x_{3}<10\right\} .
$$

Then $P(D):=D_{3}$ is surjective on $A(\Omega)$ though (2.9) is not satisfied.

Proof. The latter statement is obvious (consider $\zeta=(1,0,1 / 2)$ ). To prove surjectivity, we set

$$
g(x):=i \int_{t_{x}}^{x_{3}} f\left(x_{1}, x_{2}, \xi\right) d \xi \quad \text { for } x \in \Omega \text { and } f \in A(\Omega),
$$

where $t_{x}$ is the unique real number such that $\left.x \in \gamma\left(t_{x}\right)\right] 1 / 2,2[\times] t_{x}-1, t_{x}+1[$. Then $g$ is defined and real analytic on $\Omega$ and $D_{3} g=f$.

Example 2.12 also shows that the existence of elementary solutions defined on $\operatorname{conv}(\widehat{\omega})$ as in Corollary 2.2 rather than on $\widehat{\omega}$ as in $\left(\underline{\mathrm{B}_{\Omega}}\right)$ is also not necessary for surjectivity. In fact, let $(1,0,1 / 2),(1,0,2 \pi) \overline{\in \omega} \subset \subset \Omega$ and let $\xi:=(1,0,1-\varepsilon) \in \widehat{\omega} \subset \subset \Omega$. Then $\operatorname{conv}(\widehat{\omega})$ contains a column $C:=B_{\varepsilon}(1,0) \times[1 / 2,2 \pi]$ for large $\widehat{\omega}$. Let $F \in \mathfrak{B}(\operatorname{conv}(\widehat{\omega}))$ be as in Corollary 2.2. Then $F$ is a hyperfunction zerosolution of $D_{3}$ on $C \backslash\{\xi\}$, hence locally constant with respect to $x_{3}$. Since $F$ is real analytic near $(1,0,1 / 2)$ and 
$(1,0,2 \pi), F$ is real analytic on $U \backslash\{\xi\}$ for a neighbourhood $U$ of $\xi$. Subtracting the usual shifted elementary solution $E:=\delta_{(0,1)} \otimes \chi_{[1-\varepsilon, \infty[}$ we see in the same way that $E$ is real analytic near $\xi$ (except at $\xi$ ), a contradiction.

The methods and results from this paper are used in Langenbruch [27] to show several inheritance properties for surjectivity of partial differential operators on spaces of real analytic functions. We show that surjectivity is inherited similarly to the case of operators on $C^{\infty}$-functions. More precisely, if $P(D)$ is surjective on $A\left(\Omega_{j}\right)$ for any $j \in J$ then $P(D)$ is surjective on $A(\Omega)$ for

$$
\Omega:=\left(\bigcap_{j \in J} \Omega_{j}\right)^{\circ}
$$

and

$$
\begin{aligned}
\Omega & :=\liminf _{j}{ }^{\circ} \Omega_{j} \\
& :=\left\{\xi \in \mathbb{R}^{n} \mid \exists \varepsilon>0: B_{\varepsilon}(\xi) \subset \Omega_{j} \text { for all but finitely many } j\right\} .
\end{aligned}
$$

Also, if $P(D)$ is surjective on $A(\Omega)$, then $P(D)$ is surjective on $A\left(\Omega_{\varepsilon}\right)$ for any $\varepsilon>0$, where

$$
\Omega_{\varepsilon}:=\{\xi \in \Omega \mid \operatorname{dist}(\xi, \partial \Omega)>\varepsilon\} .
$$

As the main result of $[27]$, we obtain

THeOREm 2.13. $P(D)$ is surjective on $A\left(\mathbb{R}^{n}\right)$ if $P(D)$ is surjective on $A(\Omega)$ for some $\Omega \neq \emptyset$.

For convex $\Omega$, this is one of the main results of Hörmander [13], while the question had been open for general $\Omega$.

As already mentioned in the introduction, the heat equation was the first example of a nonsurjective partial differential operator on $A\left(\mathbb{R}^{n}\right)$ (for $n \geq 3$ ). By Theorem 2.13 we obtain the following improvement of this classical result:

EXAmPle 2.14. Let $n \geq 3$ and let $P_{m}(x):=\sum_{j=1}^{k} \partial_{j}^{2}$ for some $1<k<n$. Then there is no open set $\Omega \subset \mathbb{R}^{n}$ such that $P(D)$ is surjective on $A(\Omega)$.

Of course, this example also applies to the Schrödinger equation.

3. Necessity. In this section, we consider condition $\left(\overline{\mathrm{A}_{\Omega}}\right)$ defined as follows: For any $\omega \subset \subset \Omega$ there are $\widetilde{\omega} \subset \subset \Omega$ and $\delta>0$ such that for any $\xi \in \Omega \backslash \widetilde{\omega}$ and any $0<T \leq 1$ there are $V \in \mathfrak{U}(\Omega \times\{T\})$ and $E_{\xi} \in C_{\Delta}(W)$, $W:=V \cup(\omega \times] T-\delta, T+\delta[)$, such that

$$
P(D) E_{\xi}=\left.G(\cdot-\xi, \cdot)\right|_{W} .
$$

Clearly, $\left(\overline{\mathrm{A}_{\Omega}}\right)$ is stronger than $\left(\underline{\mathrm{A}_{\Omega}}\right)$ from the Main Theorem 2.1 and we will show in this section that $\left(\overline{\mathrm{A}_{\Omega}}\right)$ holds if $P(D)$ is surjective on $A(\Omega)$ (see Theorem 3.4). 
To this end, we will first discuss the operator $P(D)$ on $C_{\Delta}(Q)$ for a compact set $Q \subset \mathbb{R}^{n+1}$. We introduce the Grothendieck duality and condition $\left(\mathrm{C}_{\Omega}\right)$ which is equivalent to the exactness of the sequence (1.3) of spectra. Since $\left(\mathrm{C}_{\Omega}\right)$ is clearly weaker than $\left(\mathrm{A}_{\Omega}\right)$, it can also be used in the sufficiency proof in the next section. The section ends with the proof of the necessity of $\left(\overline{\mathrm{A}_{\Omega}}\right)$.

It is clear from the remarks in Section 1 that we will have to consider the operator

$$
P(D): C_{\Delta}(Q) \rightarrow C_{\Delta}(Q)
$$

for compact nonconvex sets $Q \subset \mathbb{R}^{n+1}$. We start with some corresponding auxiliary results. Since the Laplacian is elliptic, $C_{\Delta}\left(\mathbb{R}^{n+1}\right)$ is dense in $C_{\Delta}(Q)$ for any compact set $Q \subset \mathbb{R}^{n+1}$ such that

$$
\mathbb{R}^{n+1} \backslash Q \text { does not have a bounded component }
$$

(see e.g. Hörmander [14, Corollary 10.5.3]). If $L \subset Q$ is compact and also satisfies (3.1), we can thus identify $C_{\Delta}(L)^{\prime}$ with a subset of $C_{\Delta}(Q)^{\prime}$. This identification will be frequently used.

To solve the equation $P(D) f=g$ for $g \in C_{\Delta}(Q)$ by means of the HahnBanach theorem (see Remark 3.1 below) we need an appropriate representation for $C_{\Delta}(Q)_{\mathrm{b}}^{\prime}$. This is provided by the Grothendieck-Tillmann duality (which is also the reason why we have to use the canonical elementary solution $G$ of $\Delta$ in $\left(\underline{\mathrm{A}_{\Omega}}\right)$ since $G$ defines the canonical isomorphism in the duality): For $Q \subset \overline{\mathbb{R}^{n+1}}$ compact let

$$
C_{\Delta, 0}\left(\mathbb{R}^{n+1} \backslash Q\right):=\left\{f \in C_{\Delta}\left(\mathbb{R}^{n+1} \backslash Q\right) \mid \lim _{\xi \rightarrow \infty} f(\xi)=0\right\}
$$

endowed with the topology of $C\left(\mathbb{R}^{n+1} \backslash Q\right)$. Then $C_{\Delta, 0}\left(\mathbb{R}^{n+1} \backslash Q\right)$ is a Fréchet space. Furthermore,

$$
C(V \backslash Q) \text { also induces the topology of } C_{\Delta, 0}\left(\mathbb{R}^{n+1} \backslash Q\right)
$$

if $V$ is an open neighbourhood of $Q$. Let

$$
\varkappa(\mu)(x, y):=u_{\mu}(x, y):=\left\langle\mu_{(s, t)}, G(s-x, t-y)\right\rangle \quad \text { for } \mu \in C_{\Delta}(Q)_{\mathrm{b}}^{\prime} .
$$

Then we have the topological isomorphisms

$$
\varkappa: C_{\Delta}(Q)_{\mathrm{b}}^{\prime} \rightarrow C_{\Delta, 0}\left(\mathbb{R}^{n+1} \backslash Q\right) \cong C_{\Delta}\left(\mathbb{R}^{n+1} \backslash Q\right) / C_{\Delta}\left(\mathbb{R}^{n+1}\right)
$$

by the Grothendieck-Tillmann duality (Grothendieck [12, Theorem 4], Mantovani and Spagnolo [28], Tillmann [36, Satz 6]). 
A first application of (3.3) is contained in the following remark, which will be frequently used. For compact sets $Q \subset L \subset \mathbb{R}^{n+1}$ let

$$
R_{L}^{K}: C_{\Delta}(L) \rightarrow C_{\Delta}(Q)
$$

be the canonical mapping defined by restriction.

REMARK 3.1. Let $Q \subset L \subset \mathbb{R}^{n+1}$ be compact sets satisfying (3.1). Then

$$
P(D) C_{\Delta}(Q) \supset R_{L}^{Q}\left(C_{\Delta}(L)\right)
$$

if there is a neighbourhood $V$ of $L$ such that for any compact set $J \subset V \backslash L$ there is a bounded set $B \subset C_{\Delta}(Q)$ such that for any $\mu \in C_{\Delta}(Q)^{\prime}$,

$$
\sup _{(x, y) \in J}\left|u_{\mu}(x, y)\right| \leq C \sup _{f \in B}|\langle P(-D) \mu, f\rangle| .
$$

Proof. The mapping $P(-D): C_{\Delta}(Q)^{\prime} \rightarrow C_{\Delta}(Q)^{\prime}$ is injective by (3.4) and (3.3). Moreover,

$$
T:={ }^{t} R_{L}^{Q} \circ P(-D)^{-1}: H:=P(-D) C_{\Delta}(Q)^{\prime} \rightarrow C_{\Delta}(L)^{\prime}
$$

is continuous by (3.4) and (3.2) if $H$ is endowed with the topology of $C_{\Delta}(Q)^{\prime}$.

Indeed,

$$
u_{T(P(-D) \mu)}=\left.u_{\mu}\right|_{\mathbb{R}^{n+1} \backslash L}
$$

For $g \in C_{\Delta}(L)$, the mapping

$$
h: H \rightarrow \mathbb{C}, \quad P(-D) \mu \mapsto\langle\mu, g\rangle,
$$

is therefore continuous and can be extended to $\widetilde{h} \in C_{\Delta}(Q)^{\prime \prime}=C_{\Delta}(Q)$ by the Hahn-Banach theorem since $C_{\Delta}(Q)$ is a (DFS)-space and hence reflexive. Then $P(D) \widetilde{h}=R_{L}^{Q}(g)$ since

$$
\langle\mu, P(D) \widetilde{h}\rangle=\langle P(-D) \mu, \widetilde{h}\rangle=h(P(-D) \mu)=\langle\mu, g\rangle \quad \text { for } \mu \in C_{\Delta}(Q)^{\prime} .
$$

A standard estimate for $u_{\mu}$ is provided by the following basic

REMARK 3.2. Let $Q \subset \mathbb{R}^{n+1}$ be compact with (3.1). For any compact set $L \subset \mathbb{R}^{n+1} \backslash \operatorname{conv}(Q)$ there is a bounded set $B \subset C_{\Delta}(Q)$ such that for any $\mu \in C_{\Delta}\left(\mathbb{R}^{n+1}\right)^{\prime}$ with $P(-D) \mu \in C_{\Delta}(Q)^{\prime}$ we have $\mu \in C_{\Delta}(\operatorname{conv}(Q))^{\prime}$ and

$$
\sup _{(x, y) \in L}\left|u_{\mu}(x, y)\right| \leq C \sup _{f \in B}|\langle P(-D) \mu, f\rangle| \text {. }
$$

Proof. (a) By assumption, $\mu \in C_{\Delta}(J)^{\prime}$ for some compact convex set $J \subset \mathbb{R}^{n+1}$. For $w \in \mathbb{R}^{n+1}$ let

$$
V_{w}:=\left\{z \in \mathbb{R}^{n+1} \mid\langle z, w\rangle>0\right\} .
$$

Since $V_{w}$ is convex, there is $E_{w} \in C_{\Delta}\left(V_{w}\right)$ such that $P(D) E_{w}=\left.G\right|_{V_{w}}$ (as in $(2.5))$. For $z \in \mathbb{R}^{n+1}$ with $\langle z, w\rangle$ sufficiently small we get

$$
\begin{aligned}
u_{\mu}(z) & =\left\langle\mu_{\eta}, G(\eta-z)\right\rangle=\left\langle\mu_{\eta}, P(D) E_{w}(\eta-z)\right\rangle \\
& =\left\langle P(-D) \mu, E_{w}(\eta-z)\right\rangle=: v_{\mu}(z) .
\end{aligned}
$$


Then $v_{\mu}$ defines a harmonic function on

$$
W_{w, c(w)}:=\left\{z \in \mathbb{R}^{n+1} \mid\langle z, w\rangle<c(w)\right\},
$$

where $c(w):=\inf \{\langle w, \eta\rangle \mid \eta \in Q\}$. By the identity theorem, $u_{\mu}$ can thus be extended as a harmonic function on $\mathbb{R}^{n+1} \backslash \operatorname{conv}(Q)$, i.e. $\mu \in C_{\Delta}(\operatorname{conv}(Q))_{\mathrm{b}}^{\prime}$ by (3.3).

(b) For each compact convex set $L \in \mathbb{R}^{n+1}$,

$$
P(D): C_{\Delta}(L) \rightarrow C_{\Delta}(L) \text { is surjective. }
$$

Since $C_{\Delta}(L)$ is a (DFS)-space, this implies that $P(-D): C_{\Delta}(L)_{\mathrm{b}}^{\prime} \rightarrow C_{\Delta}(L)_{\mathrm{b}}^{\prime}$ is a topological isomorphism onto its range. The claim now follows from (3.3) since $C_{\Delta}(Q)_{\mathrm{b}}^{\prime}$ is continuously embedded in $C_{\Delta}(\operatorname{conv}(Q))_{\mathrm{b}}^{\prime}$.

The exactness of the sequence (1.3) of spectra can now be characterized as follows: For a compact set $K \subset \Omega$ let $R^{K}: C_{\Delta}(\Omega \times \mathbb{R}) \rightarrow C_{\Delta}(K)$ be the canonical mapping defined by restriction. Let $I(\delta):=]-\delta, \delta[$ for $\delta>0$.

Proposition 3.3. The following are equivalent:

(a) For any compact set $K \subset \Omega$,

$$
P(D) C_{\Delta}(K \times\{0\}) \supset R^{K}\left(C_{\Delta}(\Omega \times \mathbb{R})\right) .
$$

(b) The following condition $\left(\overline{\mathrm{C}_{\Omega}}\right)$ holds: for any $\omega \subset \subset \Omega$ there are $\widetilde{\omega} \subset \subset$ $\Omega$ and $\delta>0$ such that for any $\xi \in \Omega \backslash \widetilde{\omega}$ there is $E_{\xi} \in C_{\Delta}(W), W:=\omega \times I(\delta)$, such that

$$
P(D) E_{\xi}=\left.G(\cdot-\xi, \cdot)\right|_{W} .
$$

(c) The following condition $\left(\mathrm{C}_{\Omega}\right)$ holds: for any $\omega \subset \subset \Omega$ there is $\widetilde{\omega} \subset \subset \Omega$ with $\omega \subset \subset \widetilde{\omega}$ such that for any $\xi \in \partial \widetilde{\omega}$ there are $\delta_{\xi}>0$ and $E_{\xi} \in C_{\Delta}(W)$, $W:=\omega \times I\left(\delta_{\xi}\right)$, satisfying $(3.6)$.

(d) For any compact set $K \subset \Omega$ there is a compact set $J \subset \Omega$ with $K \subset J$ such that

$$
P(D) C_{\Delta}(K \times\{0\}) \supset R_{J}^{K}\left(C_{\Delta}(J \times\{0\})\right) .
$$

(e) The sequence (1.3) of projective spectra is exact.

Proof. The statements in (d) and (e) are equivalent by the definition (1.4). Similarly, (d) clearly implies (a).

(a) $\Rightarrow(\mathrm{b})$. For $K:=\bar{\omega}$ the mapping

$$
T:=P(D)^{-1} \circ R^{K}: C_{\Delta}(\Omega \times \mathbb{R}) \rightarrow C_{\Delta}(K \times\{0\}) / \operatorname{ker} P(D)
$$

is defined by (a) and continuous by the closed graph theorem. The latter space is a (DFS)-space with step spaces $Y_{k}:=B C_{\Delta}\left(\left(K+U_{1 / k}\right) \times\right.$ $I(1 / k)) / \operatorname{ker} P(D)$ of bounded harmonic functions. Thus, there is $k \in \mathbb{N}$ by Grothendieck's lemma (Meise and Vogt [30, 24.33]) such that

$$
T: C_{\Delta}(\Omega \times \mathbb{R}) \rightarrow Y_{k} \text { is continuous. }
$$


Also, there are $C, c>0$ and $\omega_{1} \subset \subset \Omega$ that

$$
\|T(f)\|_{Y_{k}} \leq C \sup _{x \in \omega_{1},|y|<c}|f(x, y)| \quad \text { for } f \in C_{\Delta}(\Omega \times \mathbb{R}) .
$$

Since $C_{\Delta}(\Omega \times \mathbb{R})$ is dense in $C_{\Delta}\left(\bar{\omega}_{1} \times \overline{I(c)}\right)$ by (3.1), $T$ can be uniquely extended to a continuous mapping $\widetilde{T}: C_{\Delta}\left(\overline{\omega_{1}} \times \overline{I(c)}\right) \rightarrow Y_{k}$ which also satisfies

$$
P(D) g=\left.f\right|_{\omega \times I(1 / k)}
$$

if $\widetilde{T}(f)=[g] \in Y_{k}$. This shows (b) for $\widetilde{\omega} \supset \supset \bar{\omega}_{1}$ since then $G(\cdot-\xi, \cdot) \in$ $C_{\Delta}\left(\bar{\omega}_{1} \times \overline{I(c)}\right)$ if $\xi \notin \widetilde{\omega}$.

(c) $\Rightarrow(\mathrm{d})$. By Remark 3.1, we have to estimate $u_{\mu}$ suitably for $\mu \in$ $C_{\Delta}(K \times\{0\})^{\prime}$. By Remark 3.2 for any compact set $L \subset \mathbb{R}^{n+1} \backslash(\operatorname{conv}(K) \times\{0\})$ there is a bounded set $B_{1} \subset C_{\Delta}(K \times\{0\})$ such that

$$
\sup _{(x, y) \in L}\left|u_{\mu}(\cdot, y)\right| \leq C \sup _{f \in B_{1}}|\langle P(-D) \mu, f\rangle| .
$$

Choose $\varepsilon>0$ such that $\omega_{1}:=K+U_{\varepsilon} \subset \subset \Omega$. Choose $\widetilde{\omega} \supset \supset \omega_{1}$ for $\omega_{1}$ by (c) and set $J:=\overline{\widetilde{\omega}}$. We may assume that $X:=\widetilde{\omega}+U_{\varepsilon} \subset \subset \Omega$. For $\xi \in \partial \widetilde{\omega}$ choose $\delta_{\xi}<\varepsilon$ and $E_{\xi} \in C_{\Delta}\left(\omega_{1} \times I\left(\delta_{\xi}\right)\right)$ by $\left(\mathrm{C}_{\Omega}\right)$. For $(x, y) \in U_{\delta_{\xi}} \times I\left(\delta_{\xi}\right)$ we then get

$$
\begin{aligned}
u_{\mu}(x+\xi, y) & =\left\langle\mu_{(s, t)}, G(s-x-\xi, t-y)\right\rangle \\
& =\left\langle\mu_{(s, t)}, P(D) E_{\xi}(s-x, t-y)\right\rangle \\
& =\left\langle P(-D) \mu_{(s, t)}, E_{\xi}(s-x, t-y)\right\rangle .
\end{aligned}
$$

Choose $\xi_{1}, \ldots, \xi_{\lambda} \in \partial \widetilde{\omega}$ such that $W:=\bigcup_{j \leq \lambda} U_{\delta_{\xi_{j}}}\left(\xi_{j}\right) \supset \partial \widetilde{\omega}$ and let $\delta:=$ $\min \left(\delta_{\xi_{1}}, \ldots, \delta_{\xi_{\lambda}}\right) / 2$. Then

$$
\sup _{(x, y) \in W \times I(\delta)}\left|u_{\mu}(x, y)\right| \leq \sup _{f \in B}|\langle P(-D) \mu, f\rangle|,
$$

where

$$
B:=\left\{E_{\xi_{j}}\left(\cdot-x-\xi_{j}, \cdot-y\right)|j \leq \lambda,| y|\leq \delta,| x-\xi_{j} \mid \leq \delta_{\xi_{j}}\right\}
$$

is bounded in $C_{\Delta}(K \times\{0\})$ since $K+U_{\delta_{\xi_{j}}} \subset \subset \omega_{1}$. The claim in (d) follows from (3.7) and (3.8) by Remark 3.1.

We now come to the main result of this section.

Theorem 3.4. Let $P(D): A(\Omega) \rightarrow A(\Omega)$ be surjective. Then $P(D)$ satisfies $\left(\overline{\mathrm{A}_{\Omega}}\right)$.

Proof. (i) Since $P(D)$ is surjective on $A(\Omega), P(D)$ satisfies $\left(\mathrm{C}_{\Omega}\right)$ and the sequence (1.3) of projective spectra is exact by Proposition 3.3. By Proposition 1.1 we therefore know that $\operatorname{Proj}^{1} N_{P}^{\Omega}=0$. 
(ii) Let $\left\{K_{k} \mid k \in \mathbb{N}\right\}$ be an increasing compact exhaustion of $\Omega$. Then $N_{P}^{\Omega}=\left(X_{k}, R_{j}^{k}\right)=\left(N_{P}\left(K_{k}\right), R_{j}^{k}\right)$ is a projective (DFS)-spectrum with

$$
X_{k}=\operatorname{limind}_{d} X_{k, d}:=\operatorname{limind}_{d} N B_{P}\left(K_{k}+V_{1 / d}\right)
$$

(see (1.7)). For $\omega \subset \subset \Omega$ choose $K_{k} \supset \supset \omega$. Theorem 1.2 of Retakh implies that there is $j \geq k$ such that

$$
R_{j}^{k}\left(N_{P}\left(K_{j} \times\{0\}\right)\right) \subset R^{k}\left(N_{P}(\Omega \times\{0\})\right)+N B_{P}\left(K_{k}+V_{1 / j}\right) .
$$

Let $K_{j} \subset \subset \omega_{1} \subset \subset \Omega$ and choose $\widetilde{\omega} \subset \subset \Omega$ and $\delta>0$ for $\omega_{1}$ by $\left(\overline{\mathrm{C}_{\Omega}}\right)$. For $\xi \in \Omega \backslash \widetilde{\omega}$ and $T>0$ the equation

$$
P(D) F=G(\cdot-\xi, \cdot)
$$

thus has solutions $f \in C_{\Delta}\left(\omega_{1} \times I(\delta)\right)$ by $\left(\overline{\mathrm{C}_{\Omega}}\right)$ and $f_{T} \in C_{\Delta}(\Omega \times\{T\})$ since $P(D)$ is surjective on $A(\Omega)$. Set

$$
g:=f-f_{T} \in N_{P}\left(K_{j} \times\{T\}\right) .
$$

By (3.9) there are $V \in \mathfrak{U}(\Omega \times\{T\}), g_{1} \in N_{P}(V), g_{2} \in N B_{P}(\omega \times] T-1 / j$, $T+1 / j[)$ and $0<\gamma<\delta_{1}:=\min (\delta, 1 / j) / 2$ such that $g=g_{1}-g_{2}$ on $\omega \times] T-\gamma, T+\gamma\left[\right.$ (since $\omega \subset K_{k}$ ). We may assume that $f_{T} \in C_{\Delta}(V)$ and $\omega \times] T-\gamma, T+\gamma\left[=V \cap(\omega \times \mathbb{R})\right.$. For $T<\delta_{1}, f_{T}+g_{1} \in C_{\Delta}(V)$ and $f+g_{2} \in C_{\Delta}(\omega \times] T-\delta_{1}, T+\delta_{1}[)$ then coincide on $V \cap(\omega \times] T-\delta_{1}$, $\left.T+\delta_{1}[)=\omega \times\right] T-\gamma, T+\gamma[$. They thus define a solution $E$ of $(3.10)$ on $V \cup(\omega \times] T-\delta_{1}, T+\delta_{1}[)$. This proves $\left(\overline{\mathrm{A}_{\Omega}}\right)$.

We will show at the end of Section 4 that $\left(\overline{\mathrm{A}_{\Omega}}\right)$ is equivalent to the surjectivity of $P(D)$ on $A(\Omega)$.

Notice that the choice of $\delta$ in $\left(\overline{\mathrm{A}_{\Omega}}\right)$ is uniform as in condition $\left(\overline{\mathrm{B}_{\Omega}}\right)$ from Theorem 2.1. However, since $T>0$ in $\left(\overline{\mathrm{A}_{\Omega}}\right)$, the functions $E_{\xi}$ coming from $\left(\overline{\mathrm{A}_{\Omega}}\right)$ are not hyperfunctions (in contrast to the elementary solutions $F_{\xi}$ from $\left.\left(\overline{\mathrm{B}_{\Omega}}\right)\right)$.

4. Sufficiency. In this section we will consider condition $\left(\underline{\mathrm{A}_{\Omega}}\right)$ from Theorem 2.1(c), i.e.: for any $\omega \subset \subset \Omega$ there is $\widetilde{\omega} \subset \subset \Omega$ with $\omega \subset \subset \widetilde{\omega}$ such that for any $\widehat{\omega} \subset \subset \Omega$ and any $\xi \in \partial \widetilde{\omega}$ there is $\delta>0$ such that for any $0<T \leq$ $\delta$ there are $V \in \mathfrak{U}(\widehat{\omega} \times\{T\})$ and $E \in C_{\Delta}(W), W:=V \cup(\omega \times] T-\delta, T+\delta[)$, such that

$$
P(D) E=\left.G(\cdot-\xi, \cdot)\right|_{W} .
$$

At the end of this section we will prove a first characterization of the surjectivity of $P(D)$ on $A(\Omega)$ including condition $\left(\mathrm{A}_{\Omega}\right)$ and part of the Main Theorem 2.1 (see Theorem 4.6). The main tool in the proof of Theorem 4.6 will be Theorem 1.5, so this section is largely devoted to verifying the assumptions of that theorem in our concrete situation. 
Our first aim is to prove that the spectrum $N_{P}^{\Omega}$ is $s w$-reduced (see Proposition 4.3 below). For this, we need two auxiliary results:

LEMMA 4.1. Let $P(D)$ satisfy $\left(\underline{\mathrm{A}_{\Omega}}\right)$.

(a) The sequence (1.3) of projective spectra is exact.

(b) $\Omega$ is $P$-convex for supports.

Proof. (a) Condition $\left(\underline{\mathrm{A}_{\Omega}}\right)$ clearly implies $\left(\mathrm{C}_{\Omega}\right)$ from Proposition 3.3. Hence, the claim follows from Proposition 3.3.

(b) This follows from (a) by Zampieri [40, Theorem 1].

LEMMA 4.2. Let $\Omega$ be $P$-convex for supports and let $P(D)$ be nonelliptic. For any $\omega \subset \subset \Omega$ there is $\widetilde{\omega} \subset \subset \Omega$ such that for any $\mu \in C_{\Delta}\left(\mathbb{R}^{n+1}\right)^{\prime}$ the following holds: If $\nu:=P(-D) \mu \in C_{\Delta}(\omega \times \mathbb{R})^{\prime}$ and if there are $\delta>0$ and a neighbourhood $Z$ of $\partial \omega$ such that $u_{\mu}$ can be extended as a harmonic function from above to $Z \times]-\delta, \infty[$ (and from below to $Z \times]-\infty, \delta[$, respectively), then $\mu \in C_{\Delta}(\widetilde{\omega} \times \mathbb{R})^{\prime}$.

Proof. The proof relies on the theory of distributional and formal boundary values of harmonic functions (see e.g. Langenbruch [20, 21]).

(i) By hypothesis we may assume that $u_{\mu}$ is defined on $V:=Z \times(\mathbb{R} \backslash\{0\})$ and that $\left.u_{\mu}\right|_{V}$ can be extended as a distribution (again denoted by $u_{\mu}$ ) to $Z \times \mathbb{R}$ such that

$$
\Delta u_{\mu}=g_{0} \otimes \delta_{y}+g_{1} \otimes \partial_{y} \delta_{y} \quad \text { on } Z \times \mathbb{R}
$$

where

$$
g_{j} \in A(Z) \text { and } P(-D) g_{j}=0 \quad \text { on } Z \text { for } j=0,1 \text {, }
$$

since we may assume that

$$
P(-D) u_{\mu}=u_{\nu} \in C_{\Delta}\left(\left(\left(\mathbb{R}^{n} \backslash \omega\right) \cup Z\right) \times \mathbb{R}\right) .
$$

(ii) We first cut off $u_{\mu}$ appropriately: Choose $\varphi \in C^{\infty}\left(\mathbb{R}^{n}\right)$ such that $\varphi=1$ on a neighbourhood $U$ of $\mathbb{R}^{n} \backslash \omega$ and $\varphi=0$ near $\omega \backslash Z$. Since $\Delta$ is elliptic, we can choose $w \in D^{\prime}((\omega \cup Z) \times \mathbb{R})$ such that

$$
\Delta w=\left(\varphi g_{0}\right) \otimes \delta_{y}+\left(\varphi g_{1}\right) \otimes \partial_{y} \delta_{y} \quad \text { on }(\omega \cup Z) \times \mathbb{R} .
$$

Then $u_{\mu}-w \in C_{\Delta}(((\omega \cup Z) \cap U) \times \mathbb{R})$ and therefore there are a compact set $Q \subset \mathbb{R}^{n+1}$ and $v \in C_{\Delta}\left(\mathbb{R}^{n+1} \backslash Q\right)$ such that

$$
v-u_{\mu} \in C_{\Delta}(U \times \mathbb{R}) \text { and } \quad v-w \in C_{\Delta}((\omega \cup Z) \times \mathbb{R}) .
$$

By (3.3) we may assume that

$$
v=u_{\lambda} \quad \text { for some } \lambda \in C_{\Delta}\left(\mathbb{R}^{n+1}\right)^{\prime} .
$$

(iii) Let $F: H\left(\mathbb{C}^{n}\right)^{2} \rightarrow C_{\Delta}\left(\mathbb{R}^{n+1}\right)$ be the canonical isomorphism defined via the solution of the Cauchy problem for $\Delta$ with data on $\mathbb{R}^{n} \times\{0\}$. Then

$$
\left\langle\varrho,\left(f_{0}, f_{1}\right)\right\rangle:=\left\langle\lambda, F\left(f_{0}, f_{1}\right)\right\rangle
$$


defines $\varrho=\left(\varrho_{0}, \varrho_{1}\right) \in\left(H\left(\mathbb{C}^{n}\right)^{\prime}\right)^{2}$ and

$$
P(-D) \varrho_{j}=P(-D)\left(\varphi g_{j}\right)=: \widetilde{g}_{j} \in C_{0}^{\infty}(\omega \backslash U) \quad \text { for } j=0,1
$$

by (4.2)-(4.6). By Fourier transformation and Malgrange's lemma (Hörmander [14, Lemma 7.3.3]) we thus have $\varrho_{j} \in C_{0}^{\infty}\left(\mathbb{R}^{n}\right)$.

With $\tilde{\lambda}:=\left(\varrho_{0} \otimes \delta_{y}+\varrho_{1} \otimes \partial_{y} \delta_{y}\right) \in C_{\Delta}\left(\mathbb{R}^{n+1}\right)^{\prime}$ we therefore get

$$
\left\langle\lambda, F\left(f_{0}, f_{1}\right)\right\rangle=\left\langle\varrho,\left(f_{0}, f_{1}\right)\right\rangle=\left\langle\widetilde{\lambda}, F\left(f_{0}, f_{1}\right)\right\rangle \quad \text { for any }\left(f_{0}, f_{1}\right) \in H\left(\mathbb{C}^{n}\right)^{2},
$$

that is, $\lambda=\widetilde{\lambda}$, and by (4.6), $v$ can be extended to $\mathbb{R}^{n+1}$ as a distribution (denoted by $v$ again) such that

$$
\Delta v=\varrho_{0} \otimes \delta_{y}+\varrho_{1} \otimes \partial_{y} \delta_{y} .
$$

This implies by (4.1) and (4.5) that

$$
\varrho_{j}=g_{j} \text { is real analytic on } Z \cap U \text {. }
$$

(iv) $\varrho_{j}$ is real analytic on $U$. Indeed, choose $\Phi \in C^{\infty}\left(\mathbb{R}^{n}\right)$ such that $\Phi=1$ on a neighbourhood $X$ of $U \backslash Z$ and $\Phi=0$ near $\omega \backslash U$. Let $H$ be a distributional elementary solution for $P(-D)$. For $x \in X$ and $a \in \mathbb{N}_{0}^{n}$ we then get

$$
\begin{aligned}
\varrho_{j}^{(a)}(x) & =\Phi(x) \varrho_{j}^{(a)}(x)=\left(H * P(-D)\left(\Phi \varrho_{j}^{(a)}\right)\right)(x) \\
& =\sum_{0<|b| \leq m}\left(H *\left(P^{(b)}(-D) \varrho_{j}^{(a)} D^{b} \Phi\right)\right)(x)(-1)^{|b|} / b !
\end{aligned}
$$

since

$$
\left(P(-D) \varrho_{j}^{(a)}\right) \Phi=\widetilde{g}_{j}^{(a)} \Phi=0
$$

by (4.7) and the choice of $\Phi$. The claim follows from (4.9) and (4.10), since supp $\operatorname{grad} \Phi \subset Z \cap U$.

(v) Since $\Omega$ is $P$-convex and $P$ is nonelliptic, $\mathbb{R}^{n+1} \backslash \Omega$ does not have a bounded component. Since $\varrho_{j}$ has compact support, we thus conclude from (iv) that supp $\varrho_{j} \subset \Omega$. Since $\Omega$ is $P$-convex and $\operatorname{supp} P(-D) \varrho_{j}=\operatorname{supp} \widetilde{g}_{j} \subset$ $\omega$, there is $\widetilde{\omega} \subset \subset \Omega$ such that $\operatorname{supp} \varrho_{j} \subset \widetilde{\omega}$. This proves the lemma by $(4.5)-(4.8)$.

Proposition 4.3. Let $P(D)$ satisfy $\left(\underline{\mathrm{A}_{\Omega}}\right)$.

(a) For any $\omega \subset \subset \Omega$ there is $\widetilde{\omega} \subset \subset \Omega$ with $\omega \subset \subset \widetilde{\omega}$ such that for any $\widehat{\omega} \subset \subset \Omega$ with $\widetilde{\omega} \subset \subset \widehat{\omega}$ there is $\beta>0$ such that for any $0<\tau<\beta$, $\left.N_{P}(\widetilde{\omega} \times I(\tau))\right|_{\omega \times I(\tau)}$ is contained in the closure of $\left.N_{P}(\widehat{\omega} \times I(\tau))\right|_{\omega \times I(\tau)}$ in $N_{P}(\omega \times I(\tau))$.

(b) $N_{P}^{\Omega}$ is sw-reduced.

Proof. (a) (I) Let $P(D)$ be elliptic and let

$$
C_{P}^{\infty}(U):=\left\{f \in C^{\infty}(U) \mid P(D) f=0\right\} .
$$


Then there is $C>0$ such that for any $\varepsilon>0$,

$$
\sup _{a}\left|f^{(a)}(0)\right| \varepsilon^{|a|} /\left(a ! C^{|a|}\right) \leq C_{1} \sup _{|x| \leq \varepsilon}|f(x)|
$$

if $f \in C_{P}^{\infty}\left(V_{\varepsilon}\right)$ is bounded.

Let

$$
\omega^{B}:=\{(x, y) \in \omega \times \mathbb{R}|| y \mid<B \operatorname{dist}(x, \partial \omega)\} .
$$

Inequality (4.11) implies that there is $B>0$ such that for any $\omega \subset \subset \mathbb{R}^{n}$,

$$
C_{P}^{\infty}(\omega)^{2}=N_{p}\left(\omega^{B}\right)
$$

where the isomorphism is provided by the solution of the Cauchy problem for $\Delta$ with data on $\omega \times\{0\}$. Notice that we have the restrictions

$$
\begin{aligned}
& \varrho: N_{p}\left(\left(\omega_{1}\right)^{B}\right) \rightarrow N_{P}(\omega \times I(\delta)) \quad \text { if } \omega \subset \subset \omega_{1} \text { and } 0<\tau<\gamma\left(\omega_{1}\right), \\
& \varrho: N_{p}(\omega \times I(\delta)) \rightarrow N_{P}\left(\omega^{B}\right)
\end{aligned}
$$

(for $\widetilde{\varrho}$ use also (4.12)). The claim thus follows in this case from the well known fact that any open set $\Omega$ is $P$-convex if $P(D)$ is elliptic, hence

$$
C_{P}^{\infty}(\Omega(1 / n)) \text { is dense in } C_{P}^{\infty}(\Omega(1 / m)) \text { if } n>m,
$$

where $\Omega(1 / k):=\left\{x \in \Omega \mid x \in U_{k}\right.$ and $\left.\operatorname{dist}(x, \partial \Omega)>1 / k\right\}$.

(II) Let $P(D)$ be nonelliptic. Let $\nu \in C_{\Delta}(\omega \times I(\tau))^{\prime}$ and let $\left.\nu\right|_{N_{p}(\widehat{\omega} \times I(\tau))}$ $=0$. We have to show that $\left.\nu\right|_{N_{p}(\widetilde{\omega} \times I(\tau))}=0$.

(i) Let $F: H\left(\mathbb{C}^{n}\right)^{2} \rightarrow C_{\Delta}\left(\mathbb{R}^{n+1}\right)$ be the canonical isomorphism defined via the solution of the Cauchy problem for $\Delta$ with data on $\mathbb{R}^{n} \times\{0\}$. Then

$$
\left\langle\widetilde{\nu},\left(f_{0}, f_{1}\right)\right\rangle:=\left\langle\nu, F\left(f_{0}, f_{1}\right)\right\rangle
$$

defines $\widetilde{\nu}=\left(\widetilde{\nu}_{0}, \widetilde{\nu}_{1}\right) \in\left(H\left(\mathbb{C}^{n}\right)^{\prime}\right)^{2}$ and $\widetilde{\nu}_{0}$ and $\widetilde{\nu}_{1}$ both vanish on the exponential solutions of $P(D)$. By Fourier transformation and Malgrange's lemma there is $\widetilde{\mu}=\left(\widetilde{\mu}_{0}, \widetilde{\mu}_{1}\right) \in\left(H\left(\left(\mathbb{C}^{n}\right)\right)^{\prime}\right)^{2}$ such that $P(-D) \widetilde{\mu}_{d}=\widetilde{\nu}_{d}$ for $d=0,1$. Then

$$
\langle\mu, f\rangle:=\left\langle\widetilde{\mu},\left(f(, 0), \partial_{y} f(, 0)\right)\right\rangle \quad \text { for } f \in C_{\Delta}\left(\mathbb{R}^{n+1}\right)
$$

defines

$$
\mu \in C_{\Delta}\left(\mathbb{R}^{n+1}\right)^{\prime} \quad \text { and } \quad P(-D) \mu=\nu .
$$

By Remark 3.2,

$$
\mu \in C_{\Delta}(\operatorname{conv}(\omega) \times I(\tau))^{\prime} .
$$

(ii) We now show that $u_{\mu}$ satisfies the assumption of Lemma 4.2.

Since $\left.Y:=\mathbb{R}^{n} \times\right] 0, \infty\left[\right.$ is convex, there is $F \in C_{\Delta}(Y)$ such that $P(D) F=$ $\left.G\right|_{Y}$ (see $\left.(2.5)\right)$. Let $\omega \subset \subset \omega_{1} \subset \subset \Omega$ and choose $\widetilde{\omega}$ for $\omega_{1}$ by $\left(\underline{\mathrm{A}_{\Omega}}\right)$. Let $\widehat{\omega} \subset \subset \Omega$ be fixed and let $\widehat{\omega} \subset \subset \widehat{\omega}_{1} \subset \subset \Omega$. For $\xi \in \partial \widetilde{\omega}$ and $\widehat{\omega}_{1}$ choose $0<\delta_{\xi}$ by $\left(\underline{\mathrm{A}_{\Omega}}\right)$. For $T_{\xi}:=\delta_{\xi} / 4$ choose $V_{\xi} \in \mathfrak{U}\left(\widehat{\omega}_{1} \times\left\{T_{\xi}\right\}\right)$ and $E_{\xi} \in C_{\Delta}\left(W_{\xi}\right)$, 
$W_{\xi}:=V_{\xi} \cup\left(\omega_{1} \times I\left(\delta_{\xi}\right)\right)$, by $\left(\underline{\mathrm{A}_{\Omega}}\right)$. Since $\widehat{\omega} \subset \subset \widehat{\omega}_{1}$ and $V_{\xi} \in \mathfrak{U}\left(\widehat{\omega}_{1} \times\left\{T_{\xi}\right\}\right)$, we may choose $0<\alpha_{\xi} \leq \delta_{\xi} / 1 \overline{6 \text { such that }}$

$$
\left(\omega+U_{\alpha_{\xi}}\right) \subset \omega_{1} \text { and }\left(\widehat{\omega}+U_{\alpha_{\xi}}\right) \times\left(T_{\xi}+I\left(2 \alpha_{\xi}\right)\right) \subset V_{\xi} .
$$

For $(x, y) \in U_{\alpha_{\xi}} \times I\left(\alpha_{\xi}\right)$ we then have

$$
\begin{aligned}
& P(D) F\left(\cdot-x-\xi, \cdot+T_{\xi}-y\right)=G\left(\cdot-x-\xi, \cdot+T_{\xi}-y\right) \\
& \quad=P(D) E_{\xi}\left(\cdot-x, \cdot+T_{\xi}-y\right) \quad \text { on } \widehat{\omega} \times I(\tau), \text { if } 0<\tau \leq \alpha_{\xi} .
\end{aligned}
$$

Hence

$$
F\left(\cdot-x-\xi, \cdot+T_{\xi}-y\right)=E_{\xi}\left(\cdot-x, \cdot+T_{\xi}-y\right)+h_{\xi, x, y}
$$

for some $h_{\xi, x, y} \in N_{P}(\widehat{\omega} \times I(\tau))$. Since $\nu\left(h_{\xi, x, y}\right)=0$ by assumption, we thus get by (4.14) and (4.15), for $(x, y) \in U_{\alpha_{\xi}} \times I\left(\alpha_{\xi}\right)$,

$$
\begin{aligned}
u_{\mu}\left(\xi+x,-T_{\xi}+y\right) & =\left\langle\mu_{(s, t)}, G\left(s-\xi-x, t+T_{\xi}-y\right)\right\rangle \\
& =\left\langle\mu_{(s, t)}, P\left(D_{s}\right) F\left(s-\xi-x, T_{\xi}-y+t\right)\right\rangle \\
& =\left\langle P(-D) \mu_{(s, t)}, F\left(s-\xi-x, T_{\xi}-y+t\right)\right\rangle \\
& =\left\langle\nu_{(s, t)}, F\left(s-\xi-x, T_{\xi}-y+t\right)\right\rangle \\
& =\left\langle\nu_{(s, t)}, E_{\xi}\left(s-x, T_{\xi}-y+t\right)\right\rangle=: v_{\xi}(x, y) .
\end{aligned}
$$

Equality (4.16) follows from (4.13), since $\mathbb{R}^{n} \times I(\tau)$ satisfies (3.1). $v_{\xi}$ is harmonic on $U:=U_{\alpha_{\xi}} \times I\left(\delta_{\xi} / 2\right)$ by (4.15), since $E_{\xi} \in C_{\Delta}\left(W_{\xi}\right)$ and $\tau \leq \alpha_{\xi} \leq$ $\delta_{\xi} / 4$. Thus $u_{\mu}$ can be extended as a harmonic function to $\left.U_{\alpha_{\xi}}(\xi) \times\right]-\infty, \delta_{\xi} / 4[$. Choose $\xi_{1}, \ldots, \xi_{d} \in \partial \widetilde{\omega}$ such that $Z:=\bigcup_{j=1}^{d} U_{\alpha_{\xi_{j}}}\left(\xi_{j}\right) \supset \partial \widetilde{\omega}$ and let

$$
\tau<\beta:=\min \left\{\alpha_{\xi_{j}} \mid j \leq d\right\} .
$$

Then

$$
\left.u_{\mu} \text { extends to } Z \times\right]-\infty, \delta[\text { (and to } Z \times]-\delta, \infty[\text { ) }
$$

from below (and from above, respectively) for $\delta:=\min \left\{\delta_{\xi_{j}} / 4 \mid j \leq d\right\}$.

(iii) Since $\Omega$ is $P$-convex for supports by Lemma 4.1(b), there is $\widetilde{\omega}_{1} \subset \subset \Omega$ by Lemma 4.2 and $(4.17)$ such that $\mu \in C_{\Delta}\left(\widetilde{\omega}_{1} \times \mathbb{R}\right)^{\prime}$. Hence $\mu \in C_{\Delta}\left(\widetilde{\omega}_{1} \times\right.$ $I(\tau))^{\prime}$ by $(4.14)$ and $P(-D) \mu=\nu$ in $C_{\Delta}\left(\widetilde{\omega}_{1} \times I(\tau)\right)^{\prime}$ by $(4.13)$ since $\widetilde{\omega}_{1} \times I(\tau)$ satisfies (3.1). Thus,

$$
\langle\nu, f\rangle=\langle P(-D) \mu, f\rangle=\langle\mu, P(D) f\rangle=0 \quad \text { for } f \in N_{P}\left(\widetilde{\omega}_{1} \times I(\tau)\right) \text { if } \tau<\beta \text {. }
$$

(b) This follows from (a) and the definition in (1.8) since $N B_{P}(\omega \times I(\tau))$ is continuously embedded in $N_{P}(\omega \times I(\tau))$ and the restriction

$$
R_{1}: N_{P}(\omega \times I(\tau)) \rightarrow N B_{P}\left(\omega_{1} \times I\left(\tau_{1}\right)\right)
$$

is continuous if $\omega_{1} \subset \subset \omega$ and $0<\tau_{1}<\tau$.

The next theorem is the crucial step in the proof of the sufficiency of $\left(\underline{\mathrm{A}_{\Omega}}\right)$, since it will give the property $\left(\mathrm{P}_{3}\right)$ from Theorem 1.5 for $N_{P}^{\Omega}$ (see the 
proof of Theorem 4.5). Let

$$
J(\delta):=[-\delta, \delta] \quad \text { for } \delta>0 .
$$

THEOREM 4.4. Let $P(D)$ satisfy $\left(\underline{\mathrm{A}_{\Omega}}\right)$.

(a) For any compact $K \subset \Omega$ there is a compact $\widetilde{K} \subset \Omega$ with $K \subset \widetilde{K}$ such that for any compact $Z \subset \Omega$ with $\widetilde{K} \subset Z$ there is $\delta>0$ such that for any $0<\gamma<\delta$ there are a compact $\widetilde{Z} \subset \Omega$ with $Z \subset \widetilde{Z}$ and $0<\beta<\gamma$ such that

$$
\begin{aligned}
P(D) C_{\Delta}((K \times J(\delta)) \cup(Z \times J(\beta))) & \\
& \left.\supset C_{\Delta}((\widetilde{K} \times J(2 \delta)) \cup(\widetilde{Z} \times J(\gamma)))\right|_{(K \times J(\delta)) \cup(Z \times J(\beta))} .
\end{aligned}
$$

(b) For any $\omega \subset \subset \Omega$ there is $\widetilde{\omega} \subset \subset \Omega$ with $\omega \subset \subset \widetilde{\omega}$ such that for any $\widehat{\omega} \subset \subset \Omega$ with $\widetilde{\omega} \subset \subset \widehat{\omega}$ there is $\delta>0$ such that for any $0<\gamma<\delta$ there are $0<\beta<\gamma$ and a continuous linear operator

$$
S: C_{\Delta}\left((\widetilde{\omega} \times \mathbb{R}) \cup\left(\mathbb{R}^{n} \times I(\gamma)\right)\right) \rightarrow C_{\Delta}((\omega \times I(\delta)) \cup(\widehat{\omega} \times I(\beta)))
$$

such that

$$
P(D) S(f)=f \quad \text { on }(\omega \times I(\delta)) \cup(\widehat{\omega} \times I(\beta)) .
$$

Proof. (a) (I) For compact $K \subset \Omega$ choose $\omega \subset \subset \Omega$ such that $K \subset \omega$. Then choose $\widetilde{\omega}$ for $\omega$ by $\left(\underline{\mathrm{A}_{\Omega}}\right)$. Choose a compact $\widetilde{K} \subset \Omega$ with $\overline{\widetilde{\omega}} \subset \operatorname{int}(\widetilde{K})$. For $Z \supset \widetilde{K}$ fixed choose $\widehat{\omega} \subset \subset \Omega$ with $\widehat{\omega} \supset Z$. We will apply Remark 3.1 for $Q:=(K \times J(\delta)) \cup(Z \times J(\beta))$ and $L:=(\widetilde{K} \times J(2 \delta)) \cup(\widetilde{Z} \times J(\gamma))$, where $\delta>\gamma>\beta>0$ and $\widetilde{Z}$ will be specified in the proof.

Let $\mu \in C_{\Delta}(Q)^{\prime}$. By Remark 3.2, for any compact $X \subset \mathbb{R}^{n+1} \backslash(\operatorname{conv}(Z) \times$ $J(\delta))$ and for any $a \in \mathbb{N}_{0}^{n}$ there is a bounded set $B \subset C_{\Delta}(Q)$ such that

$$
\sup _{(x, y) \in X}\left|D^{a} u_{\mu}(x, y)\right| \leq C \sup _{f \in B}|\langle P(-D) \mu, f\rangle| \text {. }
$$

(II) Using $\left(\underline{\mathrm{A}_{\Omega}}\right)$, we now obtain additional uniform bounds for $u_{\mu}$ in several steps.

(i) For $\xi \in \partial \widetilde{\omega}$ choose $\delta_{\xi}>0$ by $\left(\underline{\mathrm{A}_{\Omega}}\right)$. If $\delta \leq \delta_{\xi} / 2$, we may choose $V_{\xi, T} \in \mathfrak{U}(\widehat{\omega} \times\{T\})$ and $E_{\xi} \in C_{\Delta}\left(V_{\xi, T} \cup(\overline{\omega \times}] T-\delta_{\xi}, T+\delta_{\xi}[)\right)$ for $0<T \leq 2 \delta$ by $\left(\mathrm{A}_{\Omega}\right)$. Since $V_{\xi, T} \in \mathfrak{U}(\widehat{\omega} \times\{T\}), K \subset \omega$ and $Z \subset \widehat{\omega}$, there are $\varrho>0$ and $0<\overline{\nu_{\xi, T}} \leq \delta_{\xi} / 4$ such that

$$
K+\bar{U}_{\varrho} \subset \omega \text { and }\left(Z+\bar{U}_{\varrho}\right) \times\left[T-2 \nu_{\xi, T}, T+2 \nu_{\xi, T}\right] \subset V_{\xi, T} .
$$

For $(x, y) \in \bar{U}_{\varrho} \times J\left(\nu_{\xi, T}\right)$ we then get, for $a \in \mathbb{N}_{0}^{n+1}$,

$$
\begin{aligned}
D^{a} u_{\mu}(x+\xi, y-T) & =\left\langle\mu_{(s, t)}, D^{a} G(s-\xi-x, t+T-y)\right\rangle \\
& =\left\langle\mu_{(s, t)}, P\left(D_{s}\right) D^{a} E_{\xi}(s-x, t+T-y)\right\rangle \\
& =\left\langle P(-D) \mu_{(s, t)}, D^{a} E_{\xi}(s-x, t+T-y)\right\rangle
\end{aligned}
$$


if $\delta \leq \delta_{\xi} / 4$ and $\beta \leq \nu_{\xi, T}$. We may choose $\left\{\xi_{1}, \ldots, \xi_{k}\right\} \subset \partial \widetilde{\omega}$ such that

$$
\bigcup_{j \leq k} U_{\varrho}\left(\xi_{j}\right) \supset \partial \widetilde{\omega} .
$$

Set

$$
\delta:=\min \left\{\delta_{\xi_{j}} / 4 \mid j \leq k\right\} .
$$

For $j \leq k$ we may choose $T_{j, d} \in[\gamma / 2,2 \delta]$ for $d \leq \lambda(j)$ such that

$$
\left.\bigcup_{d \leq \lambda(j)}\right]-T_{j, d}-\nu_{\xi_{j}, T_{j, d}},-T_{j, d}+\nu_{\xi_{j}, T_{j, d}}[\supset[-2 \delta,-\gamma / 2]
$$

and therefore

$$
\left.V:=\bigcup_{j \leq k, d \leq \lambda(j)} U_{\varrho}\left(\xi_{j}\right) \times\right]-T_{j, d}-\nu_{\xi_{j}, T_{j, d}},-T_{j, d}+\nu_{\xi_{j}, T_{j, d}}[\supset \partial \widetilde{\omega} \times[-2 \delta,-\gamma / 2] .
$$

Let $\beta \leq \nu_{1}:=\min \left\{\nu_{\xi_{j}, T_{j, d}} \mid d \leq \lambda(j), j \leq k\right\}$. Then (4.21) shows that for any $a \in \mathbb{N}_{0}^{n+1}$,

$$
\sup _{(x, y) \in V}\left|D^{a} u_{\mu}(x, y)\right| \leq \sup _{f \in \widetilde{B}}|\langle P(-D) \mu, f\rangle|,
$$

where $\widetilde{B}:=\left\{D^{a} E_{\xi_{j}}\left(\cdot-x, \cdot+T_{j, d}-y\right)|j \leq k, d \leq \lambda(j)| x \mid, \leq \nu_{\xi_{j}, T_{j, d}}\right.$, $\left.y \in J\left(\nu_{\xi_{j}, T_{j, d}}\right)\right\}$ is bounded in $C_{\Delta}(Q)$ by (4.19). Applying (4.21) for $u_{\mu}(\cdot,-\cdot)$ instead of $u_{\mu}$, we get a bounded set $\widetilde{B} \subset C_{\Delta}(Q)$ such that for $V_{1}:=\{(x, y) \in$ $\left.\mathbb{R}^{n+1} \mid(x,|y|) \in V\right\}$,

$$
\sup _{(x, y) \in V_{1}}\left|D^{a} u_{\mu}(x, y)\right| \leq \sup _{f \in \widetilde{B}}|\langle P(-D) \mu, f\rangle| .
$$

(ii) We now decompose $u_{\mu}$ into $v_{\mu}+w_{\mu}$, keeping track of uniform estimates.

Choose a neighbourhood $U_{1}$ of $\partial \widetilde{\omega}$ such that

$$
\left.U_{1} \subset \subset \operatorname{int}(\widetilde{K}) \text { and } U_{1} \times\right] \gamma / 2,2 \delta\left[\subset V_{1}\right.
$$

and set $U:=U_{1} \cup \omega$. Choose $\varphi \in C_{0}^{\infty}(U \times I(2 \delta))$ such that $\varphi=1$ near $\overline{\widetilde{\omega}} \times J(\delta)$. Let $\widetilde{f}_{\mu}:=\left.\Delta\left(\varphi u_{\mu}\right)\right|_{W}$ for $W:=\mathbb{R}^{n} \times(\mathbb{R} \backslash J(3 \gamma / 4))$. By (4.18) and (4.21), $\widetilde{f}_{\mu}$ can be extended trivially (i.e. by taking its value equal to 0 outside $W$ ) to a bounded function $f_{\mu}$ on $\mathbb{R}^{n+1}$ such that

$$
\operatorname{supp} f_{\mu} \subset(\operatorname{supp} \operatorname{grad} \varphi) \cap \bar{W}=: \widetilde{W} \subset V_{1} \cup(\omega \times(I(2 \delta) \backslash J(\delta))) .
$$

Moreover, since $P(-D) f_{\mu}$ is the trivial extension of $P(-D) \widetilde{f}_{\mu}$ also $P(-D) f_{\mu}$ is a bounded function and

$$
\begin{aligned}
& \sup \left\{\left|P(-D) f_{\mu}(x, y)\right|+\left|f_{\mu}(x, y)\right| \mid(x, y) \in \mathbb{R}^{n+1}\right\} \\
& \leq C_{1} \sup _{\substack{(x, y) \in \widetilde{W} \\
|a| \leq m+2}}\left|D^{a} u_{\mu}(x, y)\right| \leq C_{2} \sup _{f \in B \cup \widetilde{B}}|\langle P(-D) \mu, f\rangle|
\end{aligned}
$$


by (4.22), (4.21) and (4.18). Let $g_{\mu}:=G * f_{\mu}$. Then

$$
\begin{aligned}
\sup \left\{\left|P(-D) g_{\mu}(x, y)\right|+\left|g_{\mu}(x, y)\right| \mid\right. & \left.(x, y) \in \mathbb{R}^{n+1}\right\} \\
& \leq C_{3} \sup _{f \in B \cup \widetilde{B}}|\langle P(-D) \mu, f\rangle|
\end{aligned}
$$

by (4.23). Set $v_{\mu}:=-\left.g_{\mu}\right|_{\mathbb{R}^{n+1} \backslash(\widetilde{K} \times J(2 \delta))}$ and $w_{\mu}:=(1-\varphi) u_{\mu}+\left.g_{\mu}\right|_{\mathbb{R}^{n+1} \backslash(Z \times J(\gamma))}$, where $w_{\mu}$ is defined by trivial extension of $(1-\varphi) u_{\mu}$. Then we have $v_{\mu} \in$ $C_{\Delta, 0}\left(\mathbb{R}^{n} \backslash(\widetilde{K} \times J(2 \delta))\right)$ and $w_{\mu} \in C_{\Delta, 0}\left(\mathbb{R}^{n+1} \backslash(Z \times J(\gamma))\right)$. Moreover,

$$
u_{\mu}=v_{\mu}+w_{\mu} \quad \text { on } \mathbb{R}^{n+1} \backslash L_{1}
$$

for $L_{1}:=(\widetilde{K} \times J(2 \delta)) \cup(Z \times J(\gamma))$ by the choice of $\varphi$.

(iii) Let $Y \subset \mathbb{R}^{n+1} \backslash L$ be compact. Clearly,

$$
\sup _{(x, y) \in Y}\left|v_{\mu}(x, y)\right| \leq C_{3} \sup _{f \in B \cup \widetilde{B}}|\langle P(-D) \mu, f\rangle|
$$

by (4.24). Since $w_{\mu} \in C_{\Delta, 0}\left(\mathbb{R}^{n+1} \backslash(Z \times J(\gamma))\right)$, we can find a compact $\widetilde{Z} \subset \Omega$ with $\widetilde{Z} \supset Z$ (using $\left(\underline{\mathrm{A}_{\Omega}}\right)$ for $\omega_{1} \supset Z$ as above) such that for suitable compacts $Y_{1} \subset \widetilde{Y} \subset\left(\mathbb{R}^{n} \times \overline{I(2 \gamma)}\right) \backslash(\widetilde{Z} \times J(\gamma))$ (use (3.3)), sufficiently small $\gamma>0$ and $V_{2}:=\left(V_{1} \cup \omega\right) \times \mathbb{R}$,

$$
\begin{aligned}
\sup _{(x, y) \in Y} & \left|w_{\mu}(x, y)\right| \\
\leq & C_{4} \sup _{(x, y) \in Y_{1}}\left|w_{\mu}(x, y)\right| \leq C_{5} \sup _{(x, y) \in \widetilde{Y}}\left|P(-D) w_{\mu}(x, y)\right| \\
\leq & C_{6}\left(\sup _{f \in B \cup \widetilde{B}}|\langle P(-D) \mu, f\rangle|+\sup _{(x, y) \in \widetilde{Y} \backslash V_{2}}\left|P(-D) u_{\mu}(x, y)\right|\right. \\
& \left.+\sup _{(x, y) \in \widetilde{Y} \cap V_{1}}\left|P(-D)\left(\varphi u_{\mu}\right)(x, y)\right|\right) \\
\leq & C_{7} \sup _{f \in \widehat{B}}|\langle P(-D) \mu, f\rangle|
\end{aligned}
$$

for some bounded set $\widehat{B} \subset C_{\Delta}(Q)$, where the last estimates follow from (4.24), the choice of $\varphi,(4.21)$ and (4.22). The claim in (a) now follows from (4.25)-(4.27) by Remark 3.1.

(b) Let $\omega \subset \subset \omega_{1} \subset \subset \Omega$ and set $K:=\bar{\omega}_{1}$. Choose $\widetilde{K}$ for $K$ by (a) and $\widetilde{\omega} \subset \subset \Omega$ such that $\widetilde{\omega} \supset \supset \widetilde{K}$. Fix $\widehat{\omega} \subset \subset \Omega$ with $\widehat{\omega} \supset \widetilde{\omega}$ and set $Z:=\overline{\widehat{\omega}}$. Choose $\delta>0$ by (a), fix $0<\gamma<\delta$ and choose $0<\gamma_{1}<\gamma$. Finally, choose $0<\beta<\gamma_{1}$ for $\gamma_{1}$ by (a). Then the mapping

$$
P(D)^{-1}: C_{\Delta}\left((\widetilde{\omega} \times \mathbb{R}) \cup\left(\mathbb{R}^{n} \times I(\gamma)\right)\right) \rightarrow C_{\Delta}(W) / N_{P}(W),
$$

$W:=\left(\omega_{1} \times I(\delta)\right) \cup\left(\widehat{\omega}_{1} \times I(\beta)\right)$, is defined and linear by (a). It is continuous 
by the closed graph theorem. Let

$$
L_{2, \Delta}:=L_{2}(\widetilde{W}) \cap C_{\Delta}(\widetilde{W}), \quad \widetilde{W}:=(\omega \times I(\delta / 2)) \cap(\widehat{\omega} \times I(\beta / 2)) .
$$

Then $L_{2, \Delta}$ is a Hilbert space and $N_{P, L_{2}}:=L_{2, \Delta} \cap \operatorname{ker} P(D)$ is a closed subspace. Let

$$
L: L_{2, \Delta} / N_{P, L_{2}} \rightarrow\left(N_{P, L_{2}}\right)^{\perp}
$$

be the canonical topological isomorphism. Let

$$
J: C_{\Delta}(W) / N_{P}(W) \rightarrow L_{2, \Delta} / N_{P, L_{2}}
$$

be defined by restriction. Since $J$ is continuous,

$$
S:=L \circ J \circ P(D)^{-1}: C_{\Delta}\left((\widetilde{\omega} \times \mathbb{R}) \cup\left(\mathbb{R}^{n} \times I(\gamma)\right)\right) \rightarrow C_{\Delta}(\widetilde{W})
$$

is continuous and $P(D)(S(f))=f$ on $\widetilde{W}=(\omega \times I(\delta / 2)) \cup(\widehat{\omega} \times I(\beta / 2))$.

THEOREM 4.5 (semiglobal decomposition with bounds). Let $P(D)$ satisfy $\left(\underline{\mathrm{A}_{\Omega}}\right)$.

(a) For any compact $K \subset \Omega$ there is a compact $\widetilde{K} \subset \Omega$ with $K \subset \widetilde{K}$ such that for any compact $Q \subset \Omega$ with $\widetilde{K} \subset Q$ there is $n \in \mathbb{N}$ such that for any $m \geq n$ there are $N \geq m$ and a continuous linear operator

$$
\begin{aligned}
L=\left(L_{1}, L_{2}\right): & N B_{P}\left((\widetilde{K} \times\{0\})+V_{1 / m}\right) \\
& \rightarrow N B_{P}\left((Q \times\{0\})+V_{1 / N}\right) \times N B_{P}\left((K \times\{0\})+V_{1 / n}\right)
\end{aligned}
$$

such that $L_{1}(f)+L_{2}(f)=f$ on $(K \times\{0\})+V_{1 / N}$.

(b) $N_{P}^{\Omega}$ satisfies $\left(\mathrm{P}_{3}\right)$. $\left(\mathrm{P}_{3}\right)$.

Proof. (b) This follows directly from (a) by the definitions of $N_{P}^{\Omega}$ and

(a) Notice that we have the continuous restrictions

$$
\begin{array}{ll}
\varrho: N_{P}(\omega \times I(\gamma)) \rightarrow N B_{P}\left((K \times\{0\})+V_{\delta}\right) & \text { if } \delta<\gamma \text { and } K+\bar{V}_{\delta} \subset \omega, \\
\varrho: N B_{P}\left((K \times\{0\})+V_{\delta}\right) \rightarrow N_{P}(\omega \times I(\gamma)) & \text { if } \gamma \leq \delta \text { and } \omega \subset K .
\end{array}
$$

The claim in (a) thus follows if we show the following

Claim. For any $\omega \subset \subset \Omega$ there are $\widetilde{\omega} \subset \subset \Omega$ such that for any $\widehat{\omega} \subset \subset \Omega$ there is $\delta>0$ such that for any $0<\gamma<\delta$ there are $0<\beta<\gamma$ and a continuous linear operator

$$
L=\left(L_{1}, L_{2}\right): N_{P}(\widetilde{\omega} \times I(\gamma)) \rightarrow N_{P}(\widehat{\omega} \times I(\beta)) \times N_{P}(\omega \times I(\delta))
$$

such that $L_{1}(f)+L_{2}(f)=f$ on $\omega \times I(\beta)$.

To prove the Claim, choose $\widetilde{\omega}$ for $\omega$ by Theorem 4.4(b).

(i) Let $\widetilde{\omega} \subset \subset \widetilde{\omega}_{1} \subset \subset \Omega$ and $\gamma>0$. There is a continuous linear operator

$$
R=\left(R_{1}, R_{2}\right): C_{\Delta}\left(\widetilde{\omega}_{1} \times I(\gamma)\right) \rightarrow C_{\Delta}(\widetilde{\omega} \times \mathbb{R}) \times C_{\Delta}\left(\mathbb{R}^{n} \times I(\gamma / 2)\right)
$$


such that $R_{1}(f)+R_{2}(f)=f$ on $\widetilde{\omega} \times I(\gamma / 2)$. Indeed, let $\varphi \in D\left(\widetilde{\omega}_{1}\right)$ be such that $\varphi=1$ near $\widetilde{\widetilde{\omega}}$. For $f \in C_{\Delta}\left(\widetilde{\omega}_{1} \times I(\gamma)\right)$, the function $\widetilde{f}:=\left.\Delta(\varphi f)\right|_{\mathbb{R}^{n} \times I(\gamma / 2)}$ defines a function $f_{1}$ on $\mathbb{R}^{n+1}$ (by trivial extension) which is bounded and has compact support. Then

$$
R_{1}(f):=\left.G * f_{1}\right|_{\widetilde{\omega} \times \mathbb{R}} \quad \text { and } \quad R_{2}(f):=\left.\left(\varphi f-G * f_{1}\right)\right|_{\mathbb{R}^{n} \times I(\gamma / 2)}
$$

have the required properties.

(ii) For $f \in N_{P}\left(\widetilde{\omega}_{1} \times I(\gamma)\right)$ we have $P(D) R_{1}(f)=-P(D) R_{2}(f)$ on $\widetilde{\omega} \times I(\gamma / 2)$. Thus

$$
\widetilde{R}(f):= \begin{cases}P(D) R_{1}(f) & \text { on } \widetilde{\omega} \times \mathbb{R}, \\ -P(D) R_{2}(f) & \text { on } \mathbb{R}^{n} \times I(\gamma / 2),\end{cases}
$$

defines a linear and continuous operator

$$
\widetilde{R}: N_{P}\left(\widetilde{\omega}_{1} \times I(\gamma)\right) \rightarrow C_{\Delta}(W), \quad W:=(\widetilde{\omega} \times \mathbb{R}) \cup\left(\mathbb{R}^{n} \times I(\gamma / 2)\right) .
$$

Fix $\widehat{\omega} \subset \subset \Omega$ with $\widetilde{\omega}_{1} \subset \widehat{\omega}$ and choose $\delta>0$ by Theorem $4.4(\mathrm{~b})$. Let $0<\gamma<\delta$ and choose $0<\beta$ for $\gamma / 2$ by Theorem 4.4(b). For $f \in N_{P}\left(\widetilde{\omega}_{1} \times I(\gamma)\right)$ let

$$
L_{1}(f):=\left.\left(R_{2}(f)+S \circ \widetilde{R}(f)\right)\right|_{\widehat{\omega} \times I(\beta)}, \quad L_{2}(f):=\left.\left(R_{1}(f)-S \circ \widetilde{R}(f)\right)\right|_{\omega \times I(\delta)},
$$

with the operator $S$ from Theorem 4.4(b). Then $L$ has the properties stated in the Claim for $\widetilde{\omega}_{1}$ in place of $\widetilde{\omega}$ (use also (i)).

Summarizing the results proved so far, we get the following characterization which proves the first part of the Main Theorem 2.1.

THEOREM 4.6. The following statements are equivalent:

(i) $P(D)$ is surjective on $A(\Omega)$.

(ii) For any $V \in \mathfrak{U}(\Omega)$ there is $W \in \mathfrak{U}(\Omega)$ with $W \subset V$ such that

$$
\left.P(D) C_{\Delta}(W) \supset C_{\Delta}(V)\right|_{W} .
$$

(iii) $P(D)$ satisfies $\left(\underline{\mathrm{A}_{\Omega}}\right)$.

(iv) $P(D)$ satisfies $\left(\overline{\overline{\mathrm{A}_{\Omega}}}\right)$.

Proof. (i) $\Rightarrow($ iv). This was proved in Theorem 3.4 .

(iv) $\Rightarrow$ (iii) and (ii) $\Rightarrow$ (i). These are trivial.

(iii) $\Rightarrow$ (ii). The sequence (1.3) of spectra is exact by Lemma $4.1(\mathrm{a})$. The projective (DFS)-spectrum $N_{P}^{\Omega}$ is sw-reduced by Proposition 4.3(b). Furthermore, $N_{P}^{\Omega}$ satisfies $\left(\mathrm{P}_{3}\right)$ by Theorem $4.5(\mathrm{~b})$. The claim thus follows from Theorem 1.5.

5. Regular hyperfunction elementary solutions. In this section the proof of Theorem 2.1 will be completed. The main point is to prove that the hyperfunction criterion $\left(\overline{\mathrm{B}_{\Omega}}\right)$ from Theorem 2.1(e) is necessary for the surjectivity of $P(D)$ on $A(\Omega)$. For this we have to solve the equation $P(D) f=g$ for $g \in C_{\Delta}(W)$, where $W$ is a nonconvex open set in $\mathbb{R}^{n+1}$ 
(see Theorem 5.4 below). We thus need a variant of Remark 3.1 for open sets, which is provided by the following lemma. Notice that the assumptions are stronger as compared with Remark 3.1, due to the more complicated topology of $C_{\Delta}(W)_{\mathrm{b}}^{\prime}$.

Similarly to Section 3 we will need the fact that $C_{\Delta}\left(\mathbb{R}^{n+1}\right)$ is dense in $C_{\Delta}(W)$ for the open sets $W \subset \mathbb{R}^{n+1}$ used in this section. This again follows from Hörmander [14, Corollary 10.5.3] if $W$ satisfies (3.1), i.e.

$\mathbb{R}^{n+1} \backslash W$ does not have a bounded component.

This condition holds for the open sets $W$ needed here since always

$W \cap(\{x\} \times \mathbb{R})$ is an interval for any $x \in \mathbb{R}^{n}$.

In particular, the neighbourhoods $V \in \mathfrak{U}(\Omega \times\{T\})$ can be chosen in this way (e.g. as the union of sets $\left.\omega_{j} \times\right] T-\varepsilon_{j}, T+\varepsilon_{j}[$ ).

Lemma 5.1. Let $W \subset V \subset \mathbb{R}^{n+1}$ be open sets satisfying (3.1). Then

$$
\left.P(D) C_{\Delta}(W) \supset C_{\Delta}(V)\right|_{W}
$$

if for any bounded set $B \supset C_{\Delta}(W)_{\mathrm{b}}^{\prime}$ the set

$$
\widetilde{B}:=\left\{\mu \in C_{\Delta}(V)_{\mathrm{b}}^{\prime} \mid P(-D) \mu \in B\right\} \text { is bounded in } C_{\Delta}(V)_{\mathrm{b}}^{\prime} .
$$

Proof. Let $E:=C_{\Delta}(W)_{\mathrm{b}}^{\prime}$ and $F:=C_{\Delta}(V)_{\mathrm{b}}^{\prime}$. Then (3.1) and (5.1) imply that $H:=P(-D) F \cap E$ is sequentially closed in $E$ since $F$ is a (DFS)space. Thus, $H$ is closed in $E$ (by Komatsu [19, Theorem $\left.6^{\prime}\right]$ ) since $E$ is a (DFS)-space. Therefore, $H$ is a (DFS)-space. Since $P(-D)$ is injective on $\{\mu \in F \mid P(-D) \mu \in E\}$ by (5.1),

$$
{ }^{t} R_{V}^{W} \circ P(-D)^{-1}: H \rightarrow F
$$

is defined, linear and continuous by the closed graph theorem. The proof is now completed as in Remark 3.1.

The assumptions of Lemma 5.1 will be checked in two steps: roughly speaking, we will first give bounds for the "supports" of $\mu \in C_{\Delta}(W)^{\prime}$ in terms of $\operatorname{supp}(P(-D) \mu$ ) (see Lemma 5.3, this step plays the role of $P(D)$ convexity for supports). In the second step, we then estimate $u_{\mu}$ in terms of $u_{P(-D) \mu}$. For the first step, the following remark is useful: Let

$$
\left.[\omega]_{T}:=\omega \times\right] T, \infty[\text { for } T \in \mathbb{R} .
$$

REMARK 5.2. Let $P(D)$ satisfy $\left(\overline{\mathrm{A}_{\Omega}}\right)$. For any $\omega \subset \subset \Omega$ there are $\widetilde{\omega} \subset \subset$ $\Omega$ with $\widetilde{\omega} \supset \supset \omega$ and $\delta>0$ such that for any $\xi \in \Omega \backslash \widetilde{\omega}$ and any $0<T \leq 1$ there are $F_{\xi} \in C_{\Delta}(Y), Y:=[\Omega]_{T} \cup[\omega]_{T-\delta}$, and $h_{\xi} \in C_{\Delta}(\Omega \times \mathbb{R})$ such that

$$
P(D) F_{\xi}=\left.\left(G(\cdot-\xi, \cdot)+h_{\xi}\right)\right|_{Y} .
$$

Proof. Choose $\widetilde{\omega} \subset \subset \Omega$ and $\delta>0$ by $\left(\overline{\mathrm{A}_{\Omega}}\right)$. For $\xi \in \Omega \backslash \widetilde{\omega}$ choose $V \in \mathfrak{U}(\Omega \times\{T\})$ and $E_{\xi} \in C_{\Delta}(W), W:=V \cup(\omega \times] T-\delta, T+\delta[)$, by $\left(\overline{\mathrm{A}_{\Omega}}\right)$. Take $\varphi \in C^{\infty}(\Omega \times \mathbb{R})$ such that $\varphi=1$ near $\left.\left.\Omega \times\right]-\infty, T\right]$ and $\operatorname{supp} \varphi \subset$ 
$V \cup(\Omega \times]-\infty, T])$. Then $\Delta\left(E_{\xi} \varphi\right)$ is defined on $W$ and can be extended trivially to $g \in C^{\infty}(\Omega \times \mathbb{R})$. Since $\Delta$ is elliptic, there is $g_{1} \in C^{\infty}(\Omega \times \mathbb{R})$ such that $\Delta g_{1}=g$. Moreover, $E_{\xi} \varphi$ is defined on $W$ and can be extended trivially to $E_{1} \in C^{\infty}\left(W \cup[\Omega]_{T}\right)$. Set $Y:=[\Omega]_{T} \cup[\omega]_{T-\delta}$ and $F_{\xi}:=\left.\left(E_{1}-g_{1}\right)\right|_{Y}$. Then $F_{\xi} \in C_{\Delta}(Y)$ and $P(D) F_{\xi}=\left.H_{\xi}\right|_{Y}$, where

$$
H_{\xi}:=G(\cdot-\xi, \cdot) \varphi-P(D) g_{1}+\sum_{a \neq 0} P^{(a)}(D) E_{\xi} D^{a} \varphi / a ! \in D^{\prime}(\omega \times \mathbb{R})
$$

(the last terms are again extended trivially). Since

$$
\Delta\left(P(D) F_{\xi}\right)=\Delta H_{\xi}=\delta_{(\xi, 0)}=\Delta G(\cdot-\xi, 0) \quad \text { on } \omega \times \mathbb{R},
$$

the remark is proved.

Lemma 5.3. Let $P(D)$ satisfy $\left(\overline{\mathrm{A}_{\Omega}}\right)$. Then there are a relatively compact exhaustion $\left\{\omega_{j} \mid j \in \mathbb{N}\right\}$ of $\Omega$ and a decreasing sequence $\delta_{j}>0$ such that for any decreasing sequence $0<\tau_{j}<\delta_{j}$ and any $\mu \in C_{\Delta}(\Omega \times \mathbb{R})^{\prime}$,

$$
\mu \in C_{\Delta}\left(\bigcup_{j \in \mathbb{N}}\left(\omega_{j+1} \times I\left(\tau_{j}\right)\right)\right)^{\prime} \quad \text { if } P(-D) \mu \in C_{\Delta}\left(\bigcup_{j \in \mathbb{N}}\left(\omega_{j} \times I\left(\tau_{j}\right)\right)\right)^{\prime} .
$$

Proof. With $\omega \subset \subset \Omega$ fixed set $\omega_{1}:=\omega$. For $j \in \mathbb{N}$ choose $\widetilde{\omega}=: \omega_{j+1}$ and $\delta:=\delta_{j}$ for $\omega_{j}$ by $\left(\overline{\mathrm{A}_{\Omega}}\right)$. We may assume that $\left\{\omega_{j} \mid j \in \mathbb{N}\right\}$ is a relatively compact exhaustion of $\Omega$. Let $0<\tau_{j}<\delta_{j}$ and $\mu \in C_{\Delta}(\Omega \times \mathbb{R})^{\prime}$ with $P(-D) \mu \in C_{\Delta}\left(\bigcup_{j \in \mathbb{N}}\left(\omega_{j} \times I\left(\tau_{j}\right)\right)\right)^{\prime}$. Then

$$
\mu \in C_{\Delta}(K \times J(C))^{\prime} \text { and } P(-D)\left(\bigcup_{j \leq J}\left(K_{j} \times J\left(\tau_{j}-\varepsilon_{j}\right)\right)\right)^{\prime}
$$

for suitable $C, J \in \mathbb{N}, 0<\varepsilon_{j}<\tau_{j}$ and compacts $K \subset \Omega$ and $K_{j} \subset \omega_{j}$. We may assume that

$$
K_{j}+U_{\varepsilon_{j}} \subset \omega_{j} \text { and } K+U_{\varepsilon_{1}} \subset \Omega .
$$

For $\xi \in \Omega \backslash \omega_{2}$ and $0<T \leq 1$ we choose $F_{\xi} \in C_{\Delta}\left([\Omega]_{T} \cup\left[\omega_{1}\right]_{T-\delta_{1}}\right)$ and $h_{\xi} \in C_{\Delta}(\Omega \times \mathbb{R})$ by Remark 5.2. Then we have, for $y<-C$ and $x \in U_{\varepsilon_{1}}$,

$$
\begin{aligned}
u_{\mu}(x+\xi, & y-T) \\
& =\left\langle\mu_{(s, t)}, G(s-\xi-x, t+T-y)\right\rangle \\
& =\left\langle\mu_{(s, t)}, P\left(D_{s}\right) E_{\xi}(s-x, t+T-y)-h_{\xi}(s-x, t+T-y)\right\rangle \\
& =\left\langle P(-D) \mu_{(s, t)}, E_{\xi}(s-x, T+t-y)\right\rangle-\left\langle\mu_{(s, t)}, h(s-x, t+T-y)\right\rangle \\
& :=v_{\mu}(x, y) .
\end{aligned}
$$

Now, $v_{\mu}$ is a harmonic function on $\left.U_{\varepsilon_{1}}(\xi) \times\right]-\infty,-\tau_{2}+\varepsilon_{2}[$. With the same argument applied to $u_{\mu}(\cdot,-\cdot)$ we see that $\mu \in C_{\Delta}\left(\left(\omega_{2} \times I\left(\tau_{1}\right)\right) \cup\left(\left(\Omega \backslash \omega_{2}\right) \times\right.\right.$ $\left.I\left(\tau_{2}\right)\right)^{\prime}$. As in part (a)(II)(ii) of the proof of Theorem 4.4 we now decompose 
$\mu$ as $\mu=\mu_{1}+\mu_{2}$ with $\mu_{1} \in C_{\Delta}\left(\omega_{2} \times I\left(\tau_{1}\right)\right)^{\prime}$ and $\mu_{2} \in C_{\Delta}(\Omega \times \mathbb{R})^{\prime}$, with

$$
P(-D) \mu_{2} \in C_{\Delta}\left(\bigcup_{j \geq 2} \omega_{j} \times I\left(\tau_{j}\right)\right)^{\prime} .
$$

The lemma is thus proved by applying the same argument to $\mu_{2}, \mu_{3}, \ldots$ inductively.

The hyperfunction criterion $\left(\overline{\mathrm{B}_{\Omega}}\right)$ in Theorem 2.1 will be a consequence of the result below. For $Z \subset \mathbb{R}^{n+1}$ let

$$
Z_{+}:=\{(x, y) \in Z \mid y>0\} .
$$

TheOREM 5.4. Let $P(D)$ be surjective on $A(\Omega)$. Then there is $Z \in \mathfrak{U}(\Omega)$ such that for any $\omega \subset \subset \Omega$ there are $\widetilde{\omega} \subset \subset \Omega$ with $\omega \subset \subset \widetilde{\omega}$ and $\delta>0$ such that for any $0<\widetilde{\delta} \leq \delta$,

$$
\left.P(D) C_{\Delta}\left(Z_{+} \cup(\omega \times I(\widetilde{\delta}))\right) \supset C_{\Delta}\left([\Omega]_{0} \cup[\widetilde{\omega}]_{-6 \widetilde{\delta}}\right)\right|_{Z_{+} \cup(\omega \times I(\widetilde{\delta}))} .
$$

Proof. (a) We will apply Lemma 5.1 for $W:=Z_{+} \cup(\omega \times I(\widetilde{\delta}))$ and $V:=[\Omega]_{0} \cup[\widetilde{\omega}]_{-6 \tilde{\delta}}$. Let $B \subset C_{\Delta}(W)_{\mathrm{b}}^{\prime}$ be bounded and

$$
\widetilde{B}:=\left\{\mu \in C_{\Delta}(V)^{\prime} \mid \nu:=P(-D) \mu \in B\right\} .
$$

Choose an increasing relatively compact exhaustion $\left\{\omega_{j} \mid j \in \mathbb{N}\right\}$ of $\Omega$ and a decreasing sequence $\delta_{j}>0$ by Lemma 5.3. By Theorem 4.6(ii) for $\Omega \times \mathbb{R}$ there is $X \in \mathfrak{U}(\Omega)$ such that

$$
\left.P(D) C_{\Delta}(X) \supset C_{\Delta}(\Omega \times \mathbb{R})\right|_{X} .
$$

We may assume that

$$
X=\bigcup_{j \geq 1} \omega_{j+1} \times I\left(\tau_{j}\right)
$$

for a decreasing sequence $0<\tau_{j}<\delta_{j}$. Let

$$
Z:=\bigcup_{j=1}^{\infty} \omega_{j} \times I\left(\tau_{j}\right) .
$$

We may assume that $\omega \times I(\widetilde{\delta}) \subset Z$, and therefore

$$
W:=Z_{+} \cup(\omega \times I(\widetilde{\delta}))=\bigcup_{j=1}^{\infty}\left(\omega_{j} \times\right] 0, \tau_{j}[) \cup(\omega \times I(\widetilde{\delta})) .
$$

Since

$$
B \subset C_{\Delta}(W)^{\prime} \subset C_{\Delta}(Z)^{\prime},
$$

we have $\widetilde{B} \subset C_{\Delta}(X)^{\prime}$ by Lemma 5.3 , and therefore

$$
\widetilde{B}=\left\{\mu \in C_{\Delta}(X)^{\prime} \mid \nu:=P(-D) \mu \in B\right\} .
$$


(b) There are $J \in \mathbb{N}$ and $\varrho \geq \tau_{1}$ such that

$$
\widetilde{B} \text { is bounded in } C_{\Delta}\left(\omega_{J} \times I(\varrho)\right)^{\prime} \text {. }
$$

To prove this, we will use Bonet and Galbis [5, Lemma 2]. The corresponding assumptions are easily checked: The restriction

$$
j: F:=C_{\Delta}(\Omega \times \mathbb{R}) \rightarrow E:=C_{\Delta}(X)
$$

is injective with dense range since $C_{\Delta}\left(\mathbb{R}^{n+1}\right)$ is dense in $C_{\Delta}(X)$ by $(3.1)$. Also $P(D) C_{\Delta}(\Omega \times \mathbb{R}) \subset C_{\Delta}(\Omega \times \mathbb{R})$ and $P(D) C_{\Delta}(X) \subset C_{\Delta}(X)$ are both dense since $P(D) C_{\Delta}\left(\mathbb{R}^{n+1}\right)=C_{\Delta}\left(\mathbb{R}^{n+1}\right)$. Therefore (5.2) and (5.3) imply by [5, Lemma 2$]$ that $\widetilde{B}$ is bounded in $C_{\Delta}(\Omega \times \mathbb{R})_{\mathrm{b}}^{\prime}$, since $B$ is also bounded in $C_{\Delta}(X)_{\mathrm{b}}^{\prime}$. This proves (5.4).

(c) We may assume that $\omega \subset \subset \omega_{J}$ and that $B$ is bounded in $C_{\Delta}(K)_{\mathrm{b}}^{\prime}$, where

$$
\left.K:=\bigcup_{j=1}^{J}\left(\bar{\omega}_{j} \times\left[\gamma, \tau_{j}\right]\right) \cup \overline{(\omega \times I(\widetilde{\delta})}\right)
$$

for some $0<\gamma<\tau_{J+1}$.

Let $\mu \in \widetilde{B}$ and $\omega \subset \subset \omega_{1} \subset \subset \Omega$. With $0<\beta<\min \left(\gamma, \tau_{J+1}-\gamma\right)$ to be fixed later, we now decompose $u_{\mu}$ just as in the proof of Theorem 4.4: Take $\varphi \in C_{0}^{\infty}\left(\left(\omega_{J+1} \times\right] \gamma-\beta, \varrho+1[) \cup\left(\omega_{1} \times I(2 \widetilde{\delta})\right)\right)$ such that $\varphi=1$ near $\left(\bar{\omega}_{J} \times[\gamma, \varrho]\right) \cup(\overline{\omega \times I(\widetilde{\delta})})$. Let $f_{\mu,+}$ be the trivial extension of $\widetilde{f}_{\mu,+}:=$ $\left.\Delta\left(\varphi u_{\mu}\right)\right|_{L_{+}}$, where $\left.L_{+}:=\mathbb{R}^{n} \times\right] \gamma+\beta, \infty[$. Set

$$
u_{\mu,+}:=\left.\left(G * f_{\mu,+}\right)\right|_{\mathbb{R}^{n+1} \backslash K_{+}}, \quad K_{+}:=\bar{\omega}_{J+1} \times[\gamma+\beta, \varrho+1] .
$$

Similarly, let $f_{\mu,-}$ be the trivial extension of $\widetilde{f}_{\mu,-}:=\left.\Delta\left(\varphi u_{\mu}\right)\right|_{L_{-}}$, where $L_{-}:=\left(\mathbb{R}^{n} \times\right]-\infty, \gamma+\beta[)$, and

$$
\begin{aligned}
u_{\mu,-} & :=\left.\left(G * f_{\mu,-}\right)\right|_{\mathbb{R}^{n+1} \backslash K_{-}}, \\
K_{-} & :=\left(\bar{\omega}_{J+1} \times[\gamma-\beta, \gamma+\beta]\right) \cup\left(\bar{\omega}_{1} \times[-2 \widetilde{\delta}, \gamma+\beta]\right) .
\end{aligned}
$$

Since $f_{\mu,+}$ and $f_{\mu,-}$ are bounded,

$$
u_{\mu,+} \in C_{\Delta, 0}\left(\mathbb{R}^{n+1} \backslash K_{+}\right) \text {and } u_{\mu,-} \in C_{\Delta, 0}\left(\mathbb{R}^{n+1} \backslash K_{-}\right) .
$$

Moreover,

$$
u_{\mu}=u_{\mu,+}+u_{\mu,-} \quad \text { on } \mathbb{R}^{n+1} \backslash\left(K_{+} \cup K_{-}\right),
$$

since

$$
\varphi u_{\mu}-G * f_{\mu,-}-G * f_{\mu,+} \in C_{\Delta, 0}\left(\mathbb{R}^{n+1}\right)=\{0\}
$$

by (5.5) and the relevant definitions. By (5.4) and (3.3),

$$
\left\{u_{\mu} \mid \mu \in \widetilde{B}\right\} \text { is bounded in } C_{\Delta}\left(\mathbb{R}^{n+1} \backslash\left(\overline{\omega_{J} \times I(\varrho)}\right)\right) .
$$

Therefore, by the choice of $\varphi$,

$$
\left\{f_{\mu,+} \mid \mu \in \widetilde{B}\right\} \text { is uniformly bounded, }
$$


hence

$$
\left\{u_{\mu,+} \mid \mu \in \widetilde{B}\right\} \text { is uniformly bounded in } C_{\Delta}\left(\mathbb{R}^{n+1} \backslash K_{+}\right) \text {. }
$$

Since $P(-D) u_{\mu,-}=P(-D) u_{\mu}-P(-D) u_{\mu,+}$ by $(5.6)$,

$$
\left\{P(-D) u_{\mu,-} \mid \mu \in \widetilde{B}\right\} \text { is bounded in } C_{\Delta, 0}\left(\mathbb{R}^{n+1} \backslash K_{-}\right)
$$

by (5.7) and by the fact that

$$
\{P(-D) \mu \mid \mu \in \widetilde{B}\}=B \text { is bounded in } C_{\Delta}(W)_{\mathrm{b}}^{\prime} \text { and in } C_{\Delta}\left(\overline{\left(\omega_{J} \times I(\widetilde{\delta})\right.}\right)_{\mathrm{b}}^{\prime}
$$

(the claim in the preceding line holds by assumption and (5.4)). The proof of Theorem 4.4(a) then shows that

$$
\begin{aligned}
& \left\{u_{\mu,-} \mid \mu \in \widetilde{B}\right\} \text { is bounded in } \\
& \qquad C_{\Delta}\left(\mathbb{R}^{n+1} \backslash((Q \times[\gamma / 2,3 \gamma / 2]) \cup(\widetilde{K} \times J(5 \widetilde{\delta})))\right)
\end{aligned}
$$

for suitable compacts $Q \subset \Omega$ and $\widetilde{K} \subset \Omega$, if $\beta>0$ is sufficiently small. Notice that $\delta>0$ in $\left(\overline{\mathrm{A}_{\Omega}}\right)$ only depends on $\omega$, and that $\left(\overline{\mathrm{A}_{\Omega}}\right)$ clearly also holds for $\widetilde{\delta}>0$ instead of $\delta$ if $\widetilde{\delta} \leq \delta$.

The proof of the theorem is finished by choosing $\widetilde{\omega} \subset \subset \Omega$ with $\widetilde{\omega} \supset \widetilde{K}$ and applying Lemma 5.1 and (5.6)-(5.8).

The proof of the Main Theorem 2.1 is now completed by the following result:

THEOREM 5.5. The following statements are equivalent:

(i) $P(D)$ is surjective on $A(\Omega)$.

(ii) For any $g \in A(\Omega)$ and any $\omega \subset \subset \Omega$ there is $f \in A(\omega)$ such that

$$
P(D) f=\left.g\right|_{\omega},
$$

and $P(D)$ satisfies the following condition $\left(\mathrm{B}_{\Omega}\right)$ : for any $\omega \subset \subset \Omega$ there is $\widetilde{\omega} \subset \subset \Omega$ with $\widetilde{\omega} \supset \omega$ such that for any $\widehat{\omega} \subset \subset \Omega$ and any $\xi \in \partial \widetilde{\omega}$ there is $F \in \mathfrak{B}(\widehat{\omega})$ such that

$$
P(D) F=\delta_{\xi} \quad \text { on } \widehat{\omega} \quad \text { and }\left.\quad F\right|_{\omega} \in A(\omega) .
$$

(iii) $P(D)$ satisfies (5.9) and the following condition $\left(\overline{\mathrm{B}_{\Omega}}\right)$ : for any $\omega \subset \subset$ $\Omega$ there are $\widetilde{\omega} \subset \subset \Omega$ with $\widetilde{\omega} \supset \omega$ and $\delta>0$ such that for any $\xi \in \Omega \backslash \widetilde{\omega}$ there is $F \in \mathfrak{B}(\Omega)$ such that

$$
P(D) F=\delta_{\xi} \quad \text { on } \Omega \quad \text { and }\left.\quad F\right|_{\omega} \in A_{\delta}(\omega) .
$$

(iv) $P(D)$ satisfies (5.9) and the following condition: for any $\omega \subset \subset \Omega$ there are $\widetilde{\omega} \subset \subset \Omega$ with $\widetilde{\omega} \supset \omega$ and $\delta>0$ such that for any $0<\widehat{\delta} \leq \delta$ there is $\gamma>0$ such that for any $H \in \mathfrak{B}(\Omega)$ with $\left.H\right|_{\widetilde{\omega}} \in A_{\widehat{\delta}}(\widetilde{\omega})$ there is $F \in \mathfrak{B}(\Omega)$ such that

$$
P(D) F=H \quad \text { on } \Omega \quad \text { and }\left.\quad F\right|_{\omega} \in A_{\gamma}(\omega) .
$$


Proof. (i) $\Rightarrow$ (iv). (5.9) is clearly satisfied. $P(D)$ satisfies $\left(\overline{\mathrm{A}_{\Omega}}\right)$ by Theorem 4.6. To prove the claim, we take $\omega_{1} \subset \subset \Omega$ with $\omega_{1} \supset \supset \omega$ and then choose $\widetilde{\omega}_{1}$ with $\widetilde{\omega}_{1} \supset \supset \omega_{1}$ and $\delta>0$ for $\omega_{1}$ by Theorem 5.4. Let $\widetilde{\omega}_{1} \subset \subset \widetilde{\omega} \subset \subset \Omega$ and choose $H \in \mathfrak{B}(\Omega)$ as above. Then $H$ is defined by $\widetilde{H} \in \widetilde{C}_{\Delta}(\Omega \times(\mathbb{R} \backslash\{0\}))$, and $\left.\widetilde{H}\right|_{[\Omega]_{0}}$ can be extended to $H_{1} \in C_{\Delta}\left([\Omega]_{0} \cup\left[\widetilde{\omega}_{1}\right]_{-6 \widetilde{\gamma}}\right)$ for some $0<\widetilde{\gamma} \leq \widehat{\delta}$ with $\widetilde{\gamma} \leq \delta / 6$. By Theorem 5.4 there are $Z \in \mathfrak{U}(\Omega)$ and $E_{1} \in C_{\Delta}\left(Z_{+} \cup\left(\omega_{1} \times I(\widetilde{\gamma})\right)\right)$ such that

$$
P(D) E_{1}=\left.G(\cdot-\xi, \cdot)\right|_{Z_{+} \cup\left(\omega_{1} \times I(\widetilde{\gamma})\right)} \cdot
$$

We may assume that $Z=\left\{(x, y) \mid(x,|y|) \in Z_{+}\right\} \cup(\Omega \times\{0\})$. Set $E_{2}(x, y):=$ $E_{1}(x,|y|)$ for $(x, y) \in Z \backslash(\Omega \times\{0\})$. Then $E_{2}$ satisfies (5.10) on $Z \backslash(\Omega \times\{0\})$ since $\widetilde{H}$ is even with respect to $y$. Thus, $E_{2}$ defines $F \in \mathfrak{B}(\Omega)$ with $P(D) F=$ $H$, and $\left.F\right|_{\omega} \in A_{\gamma}(\omega)$ for some $\gamma>0$, since $E_{1} \in C_{\Delta}\left(\omega_{1} \times I(\widetilde{\gamma})\right)$ and $\Delta$ is elliptic (use (4.11)).

(iv) $\Rightarrow$ (iii) $\Rightarrow$ (ii). This is trivial.

(ii) $\Rightarrow(\mathrm{i})$. By Theorem 4.6 it suffices to prove $\left(\underline{\mathrm{A}_{\Omega}}\right)$. Choose $\omega_{1} \subset \subset \Omega$ such that $\omega_{1} \supset \supset \omega$. Choose $\widetilde{\omega} \subset \subset \Omega$ for $\omega_{1}$, fix $\widehat{\omega} \subset \subset \Omega$ and choose $\widehat{\omega}_{1} \subset \subset \Omega$ with $\widehat{\omega}_{1} \supset \supset \widehat{\omega}$. For $\xi \in \partial \widetilde{\omega}$ choose $F \in \mathfrak{B}\left(\widehat{\omega}_{1}\right)$ by $\left(\underline{\mathrm{B}_{\Omega}}\right)$. Then $F$ is defined by $E_{1} \in \widetilde{C}_{\Delta}\left(\widehat{\omega}_{1} \times(\mathbb{R} \backslash\{0\})\right)$ with

$$
P(D) E_{1}=H \quad \text { on } \widehat{\omega}_{1} \times(\mathbb{R} \backslash\{0\}),
$$

where $H \in D^{\prime}\left(\widehat{\omega}_{1} \times \mathbb{R}\right)$ satisfies $\Delta H=\delta_{(\xi, 0)}$ on $\widehat{\omega}_{1} \times \mathbb{R}$. Therefore,

$$
H=G(\cdot-\xi, \cdot)+h \quad \text { for some } h \in C_{\Delta}\left(\widehat{\omega}_{1} \times \mathbb{R}\right) .
$$

For sufficiently large $\widehat{\omega}_{1}$, by (5.9), Proposition 3.3 (and extension via the Cauchy problem) and Grothendieck's lemma there is $\delta_{1}>0$ such that

$$
\left.P(-D) C_{\Delta}(\widehat{\omega} \times I(\delta)) \supset C_{\Delta}\left(\widehat{\omega}_{1} \times \mathbb{R}\right)\right|_{\widehat{\omega} \times I\left(\delta_{1}\right)} .
$$

We thus get $g \in C_{\Delta}\left(\widehat{\omega} \times I\left(\delta_{1}\right)\right)$ such that

$$
P(D) g=\left.h\right|_{\widehat{\omega} \times I\left(\delta_{1}\right)} .
$$

Since $\left.F\right|_{\omega_{1}} \in A\left(\omega_{1}\right),\left.E_{1}\right|_{[\Omega]_{0}}$ can be extended as a harmonic function (via the Cauchy problem) to $[\omega]_{-\delta_{2}}$ for some $0<\delta_{2} \leq \delta_{1}$. Equation (5.11) also holds on $[\omega]_{-\delta_{2}}$ by analytic continuation. Then

$$
E:=\left.\left(E_{1}-g\right)\right|_{W}, \quad W:=(\widehat{\omega} \times] 0, \delta_{1}[) \cup\left(\omega \times I\left(\delta_{2}\right)\right),
$$

satisfies

$$
P(D) E=G(\cdot-\xi, \cdot) \quad \text { on } W
$$

by (5.11)-(5.13). Obviously, $W \in \mathfrak{U}(\widehat{\omega} \times\{T\})$ and $W \supset \omega \times] T-\delta, T+\delta[$ for $\delta:=\delta_{2} / 2$ and $0<T \leq \delta$. This shows $\left(\underline{\mathrm{A}_{\Omega}}\right)$.

In the statement of Theorem 5.5, (5.9) can be substituted by any of the equivalent conditions from Proposition 3.3. 
By the results of this paper, it would be interesting to give precise bounds on the analytic singular support of hyperfunction elementary solutions of $P(D)$, especially for nonhomogeneous operators or operators with nonlocally hyperbolic principal part.

\section{References}

[1] K. G. Andersson, Propagation of analyticity of solutions of partial differential equations with constant coefficients, Ark. Mat. 8 (1971) 277-302.

[2] -, Global solvability of partial differential equations in the space of real analytic functions, in: Analyse fonctionnelle et applications (Rio de Janeiro, 1972), Actualités Sci. Industr. 1367, Hermann, Paris, 1975, 1-4.

[3] A. Andreotti and M. Nacinovich, Analytic Convexity and the Principle of Phragmén-Lindelöf, Scuola Norm. Sup. Pisa, 1980.

[4] G. Bengel, Das Weylsche Lemma in der Theorie der Hyperfunktionen, Math. Z. 96 (1967), 373-392.

[5] J. Bonet and A. Galbis, The range of non-surjective convolution operators on Beurling spaces, Glasgow Math. J. 38 (1996), 125-135.

[6] R. W. Braun, The surjectivity of a constant coefficient homogeneous differential operator on the real analytic functions and the geometry of its symbol, Ann. Inst. Fourier (Grenoble) 45 (1995), 223-249.

[7] R. W. Braun, R. Meise and B. A. Taylor, The geometry of analytic varieties satisfying the local Phragmén-Lindelöf condition and a geometric characterization of the partial differential operators that are surjective on $A\left(\mathbb{R}^{4}\right)$, Trans. Amer. Math. Soc., to appear.

[8] - - - - Surjectivity of constant coefficient partial differential operators on $\mathcal{A}\left(\mathbb{R}^{4}\right)$ and Whitney's $C_{4}$-cone, Bull. Soc. Roy. Sci. Liège 70 (2001), 195-206.

[9] R. W. Braun and D. Vogt, A sufficient condition for $\operatorname{Proj}^{1} \mathcal{X}=0$, Michigan Math. J. 44 (1997), 149-156.

[10] E. De Giorgi and L. Cattabriga, Una dimostrazione diretta dell'esistenza di soluzioni analitiche nel piano reale di equazioni a derivate parziali a coefficienti costanti, Boll. Un. Mat. Ital. (4) 4 (1971), 1015-1027.

[11] L. Frerick and J. Wengenroth, A sufficient condition for vanishing of the derived projective limit functor, Arch. Math. (Basel) 67 (1996), 296-301.

[12] A. Grothendieck, Sur les espaces des solutions d'une classe générale d'équations aux derivées partielles, J. Anal. Math. 2 (1952/53), 243-280.

[13] L. Hörmander, On the existence of real analytic solutions of partial differential equations with constant coefficients, Invent. Math. 21 (1973), 151-183.

[14] —, The Analysis of Linear Partial Differential Operators I, II, Grundlehren Math. Wiss. 256, 257, Springer, Berlin, 1983.

[15] A. Kaneko, On the global existence of real analytic solutions of linear partial differential equations on unbounded domain, J. Fac. Sci. Univ. Tokyo Sect. IA Math. 32 (1985), 319-372.

[16] - A sharp sufficient geometric condition for the existence of global real analytic solutions on a bounded domain, J. Math. Soc. Japan 39 (1987), 163-170.

[17] - On the global solvability of linear partial differential equations with constant coefficients in the space of real analytic functions, in: Banach Center Publ. 33, Inst. Math., Polish Acad. Sci., Warszawa, 1996, 149-160. 
[18] T. Kawai, On the global existence of real analytic solutions of linear differential equations I, J. Math. Soc. Japan 24 (1972), 481-517.

[19] H. Komatsu, Projective and injective limits of weakly compact sequences of locally convex spaces, ibid. 19 (1967), 366-383.

[20] M. Langenbruch, Randverteilungen von Nullösungen hypoelliptischer Differentialgleichungen, Manuscripta Math. 26 (1978/79), 17-35.

[21] -, P-Funktionale und Randwerte zu hypoelliptischen Differentialoperatoren, Math. Ann. 239 (1979), 55-74.

[22] - Surjective partial differential operators on spaces of ultradifferentiable functions of Roumieu type, Results Math. 29 (1996), 254-275.

[23] - Surjective partial differential operators on Gevrey classes and their localizations at infinity, Linear Topol. Spaces Complex Anal. 3 (1997), 95-111.

[24] - Localizations of partial differential operators and surjectivity on real analytic functions, Studia Math. 140 (2000), 15-40.

[25] - Surjectivity of partial differential operators in Gevrey classes and extension of regularity, Math. Nachr. 196 (1998), 103-140.

[26] - Surjective partial differential operators on real analytic functions defined on open convex sets, Manuscr. Math. 103 (2000), 241-263.

[27] —, Inheritance of surjectivity for partial differential operators on spaces of real analytic functions, preprint.

[28] F. Mantovani and S. Spagnolo, Funzionali analitici reali e funzioni armoniche, Ann. Scuola Norm. Sup. Pisa (3) 18 (1964), 475-513.

[29] A. Martineau, Sur la topologie des espaces des fonctions holomorphes, Math. Ann. 163 (1966), 62-88.

[30] R. Meise and D. Vogt, Introduction to Functional Analysis, Oxford Univ. Press, Oxford, 1997.

[31] T. Miwa, On the global existence of real analytic solutions of systems of linear differential equations with constant coefficients, Proc. Japan Acad. 49 (1973), 500502.

[32] V. P. Palamodov, The projective limit functor in the category of linear topological spaces, Math. USSR-Sb. 4 (1968), 529-558.

[33] - Homological methods in the theory of locally convex spaces, Russian Math. Surveys 26 (1971), 1-64.

[34] L. C. Piccinini, Non surjectivity of the Cauchy-Riemann operator on the space of the analytic functions on $\mathbb{R}^{n}$, Boll. Un. Mat. Ital. (4) 7 (1973), 12-28.

[35] V. S. Retakh, Subspaces of a countable inductive limit, Soviet Math. Dokl. 11 (1970), $1384-1386$.

[36] H.-G. Tillmann, Dualität in der Potential-theorie, Portugal. Math. 13 (1954), 55-86.

[37] D. Vogt, Lectures on projective spectra of (DF)-spaces, Seminar lectures, AG Funktionalanalysis, Düsseldorf/Wuppertal, 1987.

[38] —, Topics on projective spectra of (LB)-spaces, in: T. Terzioğlu (ed.), Advances in the Theory of Fréchet Spaces, NATO Adv. Sci. Inst. Ser. C 287, Kluwer, 1989, $11-27$.

[39] J. Wengenroth, Acyclic inductive spectra of Fréchet spaces, Studia Math. 120 (1996), $247-257$.

[40] G. Zampieri, A link between $C^{\infty}$ and analytic solvability for P.D.E. with constant coefficients, Rend. Sem. Mat. Univ. Padova 63 (1980), 145-150.

[41] - A sufficient condition for the existence of real analytic solutions of P.D.E. with constant coefficients, in open sets of $\mathbb{R}^{2}$, ibid., 83-87. 
[42] G. Zampieri, Propagation of singularity and existence of real analytic solutions of locally hyperbolic equations, J. Fac. Sci. Univ. Tokyo Sect. IA Math. 31 (1984), 373-390.

[43] —, Analytic solutions of P.D.E's, Ann. Univ. Ferrara Sez. VII (N.S.) 45 (1999), $365-372$.

Department of Mathematics

University of Oldenburg

D-26111 Oldenburg, Germany

E-mail: langenbruch@mathematik.uni-oldenburg.de

Received December 11, 2002

Revised version August 29, 2003 